\title{
Pre-exposure of repeated search configurations facilitates subsequent contextual cuing of visual search
}

\author{
Tom Beesley ${ }^{1}$, Miguel A. Vadillo ${ }^{2}$, D. Pearson ${ }^{1}$, and David R. Shanks ${ }^{2}$. \\ 1. University of New South Wales, Sydney, Australia \\ 2. University College London, UK
}

Running head: Pre-exposure facilitates contextual cuing

Please address correspondence to:

Dr Tom Beesley

School of Psychology

University of New South Wales

Matthews Building

Kensington Campus

Sydney, Australia.

2052

Tel: +61 (0)2 93853032

Email: t.beesley@unsw.edu.au 


\begin{abstract}
Contextual cuing is the enhancement of visual search when the configuration of distractors has been experienced previously. It has been suggested that contextual cuing relies on associative learning between the distractor locations and the target position. Four experiments examined the effect of pre-exposing configurations of consistent distractors on subsequent contextual cuing. The findings demonstrate a facilitation of subsequent cuing for pre-exposed configurations compared to novel configurations that have not been pre-exposed. This suggests that learning of repeated visual search patterns involves acquisition of not just distractor-target associations, but also associations between distractors within the search context, an effect that is not captured by the Brady and Chun (2007) connectionist model of contextual cuing. A new connectionist model of contextual cuing is proposed that learns associations between repeated distractor stimuli, enabling it to predict an effect of preexposure on contextual cuing.
\end{abstract}


The visual world we face on a daily basis is not a random collection of objects. Rather, our environment is highly structured and the tasks we conduct within it are undoubtedly facilitated by learning processes that encode the multitude of contingencies available to us. Perhaps one of the most commonly performed cognitive tasks is the process of visual search: detecting a target object within an array of distracting objects that are not the focus of our goals. For example, we may need to identify a traffic signal amongst an array of information in a busy street, and this search might be facilitated by learning that the location of the traffic signal is to the right of a particular street sign and above a bus stop shelter. Learning these contingencies allows for a rapid direction of our attention towards the possible target location based on our past experiences.

The effect of experience on repeated visual search is most clearly demonstrated in laboratory tasks examining contextual cuing (Chun \& Jiang, 1998). In a typical experiment participants are given instructions that the task measures visual search performance, but surreptitiously a set of search configurations (the organization of the distractor stimuli and the target position) are repeated across the course of the experiment. Intermixed with these trials participants are presented with random non-repeated search configurations (hereafter random configurations) that do not repeat again during the experiment. Reaction times (hereafter RTs) on repeated search trials are typically considerably faster than those on random configurations, indicating that participants learn the contingent relationships between distractor locations and target position; these effects are robust and have now been shown in numerous research laboratories across many different types of stimuli and conditions (e.g., Beesley \& Shanks, 2012; Jiang \& Wagner, 2004; Jiménez \& Vázquez, 2011; Kunar, Flusberg, Horowitz, \& Wolfe, 2007; Olson \& Chun, 2002).

There is still a great deal of debate as to the mechanisms responsible for even the most fundamental aspects of performance in the contextual cuing task. Current debate has focussed 
on the question of whether learning reflects a facilitation of attention to the target location (Chun \& Jiang, 1998) or a facilitation in target detection (e.g., Kunar, Flusberg, Horowitz, \& Wolfe, 2007). What is clear, however, is that participants are learning to associate the spatial arrangement of distractor locations with the spatial location of the target and whilst the debate continues as to how this learning manifests itself in search performance (enhanced localization or enhanced detection), the present article examines the form that these associative representations take in memory. Returning to our initial example, the current article asks: what is learnt about the position of the traffic signal amongst the street sign and the bus stop that leads to facilitated cuing of the location of the traffic signal in the future?

One possibility is that during contextual cuing associations form only between the individual distractor objects and the target object of the search. The most formal description of this account was provided by Brady and Chun (2007) who proposed an associative model of contextual cuing that forms direct associations from distractor locations to target locations. In this model, associative learning takes place on a distractor-by-distractor basis, such that co-occurring distractors acquire independent connections to the target and can prime the target location independently of other associations that have been formed. The model suggests that contextual cuing is competitive, such that existing associations will modulate the learning of novel but redundant associations (although see Beesley \& Shanks, 2012, for a demonstration that such competition may not occur in contextual cuing).

There is a great deal of supporting evidence that the learning of distractor-target associations is a key component of contextual cuing. For example, the contextual cuing effect is reduced or even abolished when the target position is relocated in a second phase of the experiment (Manginelli \& Pollmann, 2009; Makovski \& Jiang, 2010). Potential target stimuli also appear to be afforded special encoding in the memory representation of stored contexts. Conci, Sun, and Müller (2011; see also Conci \& Müller, 2012) used a task in which contexts 
were trained with two target stimuli present on a given trial, one consistent with the designated task response and a second target that was initially inconsistent. When the second target became relevant, contextual cuing was still observed (albeit somewhat reduced). These data clearly support the learning of direct associations between distractor stimuli and target stimuli in the task.

The current article examines whether learning over-and-above that of the individual distractor-target associations takes place when we learn about repeated search contexts. In our example, we might ask whether searching for a shop sign is facilitated when carried out in the same traffic scene that we are already familiar with as a result of searching for the traffic signal. This seems plausible: when we search for different objects within a scene it would seem inefficient to encode the search context anew for every combination of searchcontext and target. Perhaps more likely is that the process of visual search for one object may lead (at least in part) to a representation forming for the search configuration as a whole. Importantly, such an effect is not anticipated by any account that describes contextual cuing as resulting only from the formation of associations between distractor and target stimuli. For example, according to Brady and Chun's (2007) model, associations that form as a result of consistent searches in fixed contexts (contextual cuing) would lead to an impairment in contextual cuing for subsequent searches at a different location. The model would effectively need to extinguish the established distractor-target associations and relearn new distractortarget associations for these new contingencies. Indeed, there are several demonstrations of the severe inflexibility of the learning system to such changes in distractor-target contingencies. Zellin, Conci, von Mühlenen, and Müller (2013) extended the design of Manginelli and Pollmann (2009) in which targets were remapped to new positions, replicating the abolition of contextual cuing, but also demonstrating that a return of the target to its originally trained position sparked an immediate recovery of contextual cuing. 
Furthermore, the impact and resilience of this proactive interference on the original distractor-target associations was observed even over extended periods of training. Again, these results suggest that contextual cuing is driven by the initial encoding of associations between distractor locations and the initial target position.

In this article we explore whether, in addition to distractor-target associations, contextual cuing may also involve the learning of distractor-distractor associations. Since contextual cuing experiments have tended to train consistent distractor-target associations, in many cases it has not been possible to conclude whether such learning occurs in contextual cuing. To our knowledge only a few studies have directly sought evidence for distractordistractor (or configural) learning in contextual cuing. Of particular interest is a study by Jiang and Wagner (2004, Experiment 1; see also Olson \& Chun, 2002; Song \& Jiang, 2005), who trained participants in a first stage with repeated patterns of context in a standard design, before, in a transfer test, combining subsets of distractors from two contexts that had been paired with the same target. If learning of distractor-distractor associations is crucial for contextual cuing, then we would expect such recombination of contexts to disrupt the contextual cuing effect. However, Jiang and Wagner observed contextual cuing effects of a similar magnitude for the trained contexts and the recombined contexts. We note however, that recombined displays were constructed from two separate half-patterns in the transfer phase and so a significant proportion of the configural information was retained in the transfer displays. In a second experiment, Jiang and Wagner examined the contextual cuing elicited by transformations on the originally trained contexts that preserved the original configurations of distractors (distractor positions were rescaled and uniformly displaced in one direction). In these transformed displays, the locations of individual distractors were all changed and hence if contextual cuing depends solely on relationships between individual distractors and the target, cuing should be disrupted. It was found that contextual cuing was 
unimpaired by such transformations, providing some support for the role of configural or distractor-distractor representations in contextual cuing (although see Brady \& Chun, 2007, for a discussion of how their elemental theory may account for this performance).

In the present experiments we provide a systematic examination of whether contextual cuing results in the learning of distractor configurations independently of distractor-target associations. In all experiments we train a set of configurations with a pseudo-randomly placed target on each trial. We anticipated that this pre-exposure period would allow for the learning of consistent configurations of distractors in the absence of a consistent distractor-target association. In a subsequent phase we train a new configurationtarget association (the configurations become predictive of one particular target location) for these previously exposed configurations and compare the contextual cuing that occurs to that for novel repeated configurations. It is important to note that we do not expect that the repetition of non-predictive configurations will produce a contextual cuing effect in and of itself. In fact, Chun and Jiang (Experiment 3, 1998) showed that such repetition of contexts does not produce a contextual cuing effect. Rather the suggestion is that this repetition may result in the encoding of a representation of the search context, leading to enhanced learning of subsequent consistent target placements at a later stage.

\section{Experiment 1}

Experiment 1 aimed to examine the effect that pre-exposure to non-predictive repeated configurations has on subsequent contextual cuing. This was examined by pairing the preexposed configurations with consistent target-positions in the subsequent training phase and comparing search efficiency on these configurations to novel repeated configurations. Given the pre-exposure of non-predictive configurations in the first phase, the experiment also 
provides a conceptual replication of Chun and Jiang's (Experiment 3, 1998) demonstration of equivalent search times to repeated non-predictive and random configurations.

\section{Method}

Participants. Twenty participants from the University of New South Wales participated in exchange for course credit. The experiment lasted for approximately 25 minutes.

Apparatus. The experiment was conducted on PCs with 19" TFT monitors set at a resolution of 1024 x 768. Stimulus presentation and response recording was handled by software programmed using MatLab, Cogent 2000 and Cogent Graphics (www.vislab.ucl.ac.uk/cogent.php). The experiment was programmed by T. Beesley (whereas subsequent experiments were programmed by M. A. Vadillo). Stimuli were drawn within the experiment software. Distractor stimuli were letter Ls and the target stimulus was a letter T. The letter stimuli were $13 \mathrm{~mm}$ square. For the distractors, the vertical line of the letter L was offset slightly (less than $1 \mathrm{~mm}$ ) from the end of the horizontal line in order to increase the similarity between distractor and target shapes and therefore increase the difficulty of the visual search task.

Stimuli were arranged in an invisible square grid of 144 evenly spaced cells (12 x 12) which was positioned centrally on the screen and was $240 \mathrm{~mm}$ square. The fixation cross (displayed centrally before each trial) was $11 \mathrm{~mm}$ square. Responses to the target stimulus were made with keys $\mathrm{z}$ and $\mathrm{m}$ on a standard PC keyboard. The background color of the screen was grey. Stimuli were colored blue, red, green or yellow. Distractor stimuli were oriented by rotating the letter $\mathrm{L}$ by $0^{\circ}, 90^{\circ}, 180^{\circ}$, or $270^{\circ}$. Target stimuli were oriented by rotating the letter $\mathrm{T}$ by $90^{\circ}$ or $270^{\circ}$. The color and orientation of the distractors were randomly assigned for each pattern, with the constraint that there could be no more than eight distractors of one color, and at least two distractors of each color. Repeated elements of patterns maintained the 
same color and orientation for distractor stimuli across repetitions. Target color was also randomly determined and maintained across presentations of the same pattern, but target orientation was determined randomly within each block of trials.

Design. Patterns consisted of 17 stimuli (16 distractors plus 1 target) with four distractors placed in each quadrant of the pattern. Eight target locations were used in total, two in each quadrant of the screen. Target positions were chosen at random from one of five locations within each quadrant that were approximately equidistant from the centre of the screen. Distractors could not appear in these target locations.

In the first phase of the experiment participants were trained with four repeated configurations of distractors. In all experiments reported here, four configurations were trained for each trial type. This method has two advantages. Firstly, it has been suggested that contextual cuing effects are driven by learning of 1 or 2 configurations (Smyth \& Shanks, 2008) and that training fewer repeated configurations leads to more robust cuing effects (Bennett, Barnes, Howard, \& Howard, 2009). Thus training only a small number of configurations reduces unwanted noise in any measure of contextual cuing. Secondly, four configurations allows for the placement of the target in four quadrants of the screen, ensuring spatial frequency effects for targets are minimised (see Jiang, Swallow, \& Rosenbaum, 2013). Each of these configurations was presented four times in each block of this phase and, importantly, within this block each configuration was presented with each of four target locations assigned to the repeated configurations. Thus, while the configuration of distractors repeated across trials, it could not be used as a valid cue for target location. Random configurations were trained alongside repeated configurations during this phase. These two sets of four configurations (repeated and random) were each assigned four target locations, one from each quadrant of the display. 
In the second phase of the experiment participants were presented with the preexposed configurations of distractors as well as a novel set of repeated configurations. Importantly, in this phase all configurations were predictive of a specific target location. This was achieved by pairing one configuration within each set with one target location in each quadrant of the display. In order to minimize the effect of any residual associations between repeated target locations and any of the four target locations they were presented with in the first phase, the target sets were switched for pre-exposed repeated configurations. That is, the target locations used for repeated configurations in the first stage were used for the novel configurations in the second phase, while the target locations used for random configurations in the first stage were used for pre-exposed repeated configurations in the second phase.

Procedure. At the start of the experiment participants received instructions detailing the nature of the visual search task. Example displays were presented and participants were shown the correct response for each orientation of the target.

The first phase of the experiment consisted of 5 blocks of 32 trials. Each block contained four presentations of each repeated configuration with each of the four targets, as well as 16 random configurations. The second phase of the experiment consisted of 10 blocks of 8 trials, with each of the four pre-exposed repeated configurations presented once and each of the four novel repeated configurations presented once.

Within each block, trials were presented in a random order with the constraint that consecutive trials across adjoining blocks could not present the same repeated pattern. Any given target position could not occur on consecutive trials. Target orientation (left or right) was determined randomly, but with an equal number of presentations of each orientation within a sub-block of 8 trials. 
Each trial commenced with a fixation cross presented in the centre of the screen for $1000 \mathrm{~ms}$, which was then replaced immediately by the pattern of stimuli. RTs were recorded from the onset of the pattern. Following a valid response $(\mathrm{z}$ or $\mathrm{m})$ the pattern was removed from the screen. The response-stimulus interval (hereafter RSI) was $1000 \mathrm{~ms}$. If participants made an incorrect response to the target orientation, "ERROR!" appeared in the centre of the screen for $2000 \mathrm{~ms}$, prior to the RSI. A rest-break of 20 seconds was given every 108 trials. Trials started automatically after these breaks. The transition between the pre-exposure and training phases was not signalled to participants.

\section{Results}

All participants performed the task with a high degree of accuracy: mean $99.4 \%$. Two participants produced mean RTs two standard deviations (hereafter SDs) greater than the mean of the sample and were removed as outliers. Trials on which an incorrect response was made, trials immediately after a rest-break and trials longer than 10 seconds were all discounted from the analysis of RTs. RTs in this and all subsequent experiments were natural $\log$ transformed and those RTs longer than 2 SDs above or below the mean RT were discounted. All analyses were conducted on natural log transformed data, though nontransformed data are presented in the figures.

The data from the pre-exposure phase are presented in Figure 1A. As expected, search times to (non-predictive) repeated configurations and random configurations were equivalent across the course of this phase. This was confirmed by a two-way repeated measures ANOVA with factors of configuration (repeated vs. random) and block, which revealed a main effect of block, $F(4,68)=19.92, p<.001$, but no main effect of configuration, $F(1,17)=$ $1.23, p=.28$, nor an interaction between configuration and block, $F(4,68)=1.04, p=.39$. 
Of particular interest are the data from the training phase which are presented in Figure 1B. Search times decreased rapidly across the block with a clear tendency for faster search times on the pre-exposed repeated configurations. This was confirmed by a two-way ANOVA with factors of configuration (pre-exposed repeated vs. repeated) and block, which revealed a main effect of configuration, $F(1,17)=6.53, p<.05$, and block, $F(9,153)=2.79, p$ $<.01$, but no interaction between these factors, $F<1$.

\section{Discussion}

In Experiment 1, participants were trained with a pre-exposure phase in which a set of four configurations were presented but were not predictive of target location. The results from this phase represent a replication of Chun and Jiang's (1998) Experiment 3: contextual cuing did not occur for repeated configurations trained with targets placed in inconsistent locations. However, Experiment 1 sought to examine whether this pre-exposure of repeated configurations conferred any benefit to contextual cuing with these patterns in a subsequent stage. Indeed the pre-exposure phase had exactly this consequence: when the pre-exposed configurations were subsequently trained with consistent target locations, a stronger contextual cuing effect was observed compared to that for a comparable novel set of repeated configurations.

The results suggest that despite the fact that the configurations were inconsistently paired with target placements in the first phase, representations of the pre-exposed configurations were formed that later facilitated target search. One possibility is that the representation that is stored during pre-exposure acts as a more salient cue for the contextual cuing that develops in the second phase, leading to stronger associations with the target location. Our procedure will presumably have resulted in an absence of any reliable 
distractor-target pairings during the pre-exposure phase, and will also have ensured that these associations would be unable to contribute to cuing in the second phase. As such, the results of Experiment 1 are difficult to account for by a purely elemental model of contextual cuing (such as that provided by Brady \& Chun, 2007). Instead the results suggest that contextual cuing is driven, at least in part, by the learning of distractor-distractor associations or the formation of configural representations during pre-exposure.

\section{Experiment 2}

In Experiment 1 repeated configurations were pre-exposed with inconsistent target locations in a standard visual search task. Our account of this effect centres on the encoding of associations within the distractor configuration itself. The placement of the target position across all four quadrants of the display during pre-exposure meant it was rather unlikely that the pre-exposure advantage stemmed from learning distractor-target associations during the pre-exposure phase. While the interaction effect was not significant, the facilitation in cuing for pre-exposed configurations did not appear to transfer directly from pre-exposure, since search times on pre-exposed and novel repeated configurations were equivalent in the first block of the training phase (see Figure 1B). Following this initial block, a clear difference was observed in the response times to pre-exposed and novel repeated configurations.

If the pre-exposure effect does not result from learning associations between distractors and the target position, it follows that the pre-exposure effect should be observed even when the configuration of distractors is pre-exposed without a target present on each trial. Thus, in Experiment 2 participants experienced configurations on both target-present and target-absent trials during the pre-exposure phase, before receiving a standard contextual cuing task consisting only of target-present trials. Importantly, we pre-exposed two sets of 
configurations, one set presented only on target-present trials during the pre-exposure phase and one set presented only on target-absent trials in the pre-exposure phase. As in Experiment 1 these configurations were intermixed with random configurations for both target-present and target-absent trials. In a subsequent stage both sets of configurations were trained with consistent target locations to examine contextual cuing. We again included a novel set of repeated configurations against which we could assess the rate of learning. We also included random configurations in this phase allowing us to assess the extent of learning for each configuration type.

\section{Method}

Participants. Fifty-two participants from University College London participated in the experiment. Participants were paid $£ 4$ with a penalty imposed of $2 p$ for every incorrect response or for every response slower than 3 seconds. The experiment lasted for approximately 35 minutes.

Apparatus. The experiment was conducted on PCs with 17" TFT monitors set at a resolution of 1280 x 1024 . The experiment was programmed by M. A. Vadillo and was similar to Experiment 1 except for the following minor differences. The letter stimuli were $14 \mathrm{~mm}$ square. Unlike in Experiment 1, the vertical line of the letter L was not offset from the end of the horizontal line. This made the visual search task marginally easier than Experiment 1 by making distractors less similar to the target. The stimuli formed a $270 \mathrm{~mm}$ invisible square grid. The fixation cross was $8 \mathrm{~mm}$ square. For no-target trials participants issued a "targetabsent response" with the spacebar. 
Design. Patterns consisted of 17 stimuli (16 distractors plus 1 target) with four distractors placed in each quadrant of the pattern. In the first phase of the experiment participants were trained with eight repeated configurations of distractors, four in the "targetpresent" set and four in the "target-absent" set. For the target-present set, each configuration was presented an equal number of times with a target in each of the four quadrants of the screen. During this stage, the target could appear in any randomly chosen location of the screen, with the only constraint that for any given repeated pattern, the target could not appear again in one quadrant until it had already appeared in all the other quadrants. Additionally, the target could appear in any color. Therefore, as in Experiment 1, while the configuration of distractors repeated across trials, it could not be used as a valid cue for target location. For the target-absent set, the four configurations were presented an equal number of times but without target stimuli. Random configurations of both target-present and targetabsent forms were intermixed with repeated configurations during this phase.

In the second phase of the experiment participants were presented with both sets of pre-exposed configurations of distractors as well as a new set of repeated configurations. All configurations were of the target-present type and were predictive of a specific target location. During this phase sixteen target locations were used, four in each quadrant of the screen. Target positions were chosen at random from a circle of locations approximately equidistant from the centre of the screen. Distractors could not appear in these target locations. Four different sets of four targets were selected and used for the four different sets of configurations: repeated pre-exposed (target-present); repeated pre-exposed (targetabsent); repeated (novel); and random. Each set contained one target from each quadrant of the display.

Procedure. The procedure was similar to Experiment 1 with the exception of the following changes. Participants were instructed to use their index fingers for keys $\mathrm{z}$ and $\mathrm{m}$ 
and to signal a "target-absent response" by pressing the spacebar with the thumb of their dominant hand.

The first phase of the experiment consisted of 24 blocks of 16 trials. Each block contained one presentation of each of the four repeated configurations from both the targetpresent and target absent sets, as well as four random target-present configurations and four random target-absent configurations. The second phase of the experiment consisted of 12 blocks of 16 trials, with configurations from both sets of pre-exposed repeated configurations presented once and each of the four novel repeated configurations presented once, along with four random configurations. All configurations were of type target-present during the second phase.

Unlike in Experiment 1, in the present experiment the target orientation (left or right) was not constrained to an equal number of left/right presentations within sub-blocks; the target orientation was decided randomly on a trial-by-trial basis. Participants searched for the target and responded with keys $\mathrm{z}$ or $\mathrm{m}$ for detection of the target (according to its orientation) or with the spacebar if the target was absent. A rest-break of 20 seconds was given every 100 trials.

\section{Results}

Two participants produced error rates on target-present trials that were greater than two standard deviations above the mean error rate of the sample, and three additional participants produced mean RTs that were more than two standard deviations above the mean of the sample. These five participants were not included in the final analysis. The criteria used in Experiment 1 were applied for the exclusion of long RTs and erroneous trials, with outliers 
identified separately for target-present and target-absent trials (given the clear difference in RTs on these trials, see below). Data were combined into two-block averages for purposes of data presentation and analysis.

Figure 2A shows the data for the pre-exposure phase. Clearly search times were much faster on target-present trials than on target-absent trials. This is likely to be due to a targetabsent trial requiring an exhaustive search, whereas a target-present search can be terminated, on average, after inspecting half the items. A three-way repeated measures ANOVA with factors of configuration (repeated vs. random), target (present vs. absent), and block revealed a main effect of configuration, a main effect of target, and a main effect of block, all $F_{\mathrm{s}}>$ 28.3 all $p s<.001$. Interestingly there was an interaction between configuration and target, $F(1,46)=6.41, p<.05$, indicating that the difference in response times between repeated and random configurations was greater in the case of the target-absent trials. There were significant interaction effects between configuration and block, and target and block, $F \mathrm{~s}>$ $2.09, p \mathrm{~s}<.05$; the three-way interaction effect was not significant, $F(11,506)=1.22, p=.27$. In order to understand the significant interaction between target and configuration separate $t$ tests were conducted on target-present and target-absent data to explore the difference between responses to pre-exposed and random configurations. This revealed that RTs to preexposed configurations were faster than those to random configurations on the target-absent trials, $t(46)=7.11, p<.001$, but not on the target-present trials, $t(46)=1.50, p=.14$.

Figure 2B shows the data for the training phase. As in Experiment 1, it appears that contextual cuing was stronger for pre-exposed contexts, but that this effect is limited to those contexts trained with targets in the first phase. These data were subjected to a repeated measures ANOVA with factors configuration (pre-exposed target-present repeated, preexposed target-absent repeated, novel repeated, and random) and block. This revealed a main effect of configuration, $F(3,138)=6.40, p<.001$, a main effect of block, $F(5,230)=11.25, p$ 
$<.001$, but no interaction between these factors, $F(15,690)=1.42, p=.13$. Follow up planned comparisons revealed that responding on the novel repeated configurations was faster than responding on random configurations, $F(1,46)=24.73, p<.001$, demonstrating a contextual cuing effect in the training phase. Responses to configurations pre-exposed on target-present trials were faster than to novel repeated configurations, $F(1,46)=5.19, p<.05$, replicating the results of Experiment 1, but there was no difference between responses to configurations pre-exposed on target-absent trials and novel repeated configurations, $F<1$. Finally responses to configurations pre-exposed on target-present trials were faster than responses to configurations pre-exposed on target-absent trials, $F(1,44)=4.88, p<.05$.

Although the interaction between configuration and block was not significant, it is of particular interest to examine whether the contextual cuing effect for pre-exposed target present configurations developed during the training phase or was present immediately on transfer to the training phase. An analysis on the first block of trials found that over the first 16 trials within that block there was no contextual cuing effect (RTs to repeated configurations $=1022 ;$ RTs to random configurations $=1033), t<1$, but a contextual cuing effect was present over the second set of 16 trials in block 1 (RTs to repeated configurations $=953$; RTs to random configurations $=1054), t(46)=2.84, p<.01$. Thus, whilst there was a rapid development of contextual cuing for the pre-exposed target-present configurations at the start of the training phase, it was not present at the immediate outset of this training phase.

\section{Discussion}

In Experiment 2 participants were trained initially with repeated but non-predictive configurations across two different types of search trial: those with targets present and those 
without targets. There was a clear contextual cuing effect on the target-absent trials, but as in Experiment 1 this was not observed in the target-present trials. We note that this particular finding for the target-absent trials is inconsistent with published data from Kunar and Wolfe (2011); we discuss this discrepancy, along with the similar finding from Experiment 4, in the General Discussion.

Following pre-exposure participants received a standard contextual cuing task (targetpresent trials throughout) in which pre-exposed configurations were trained as predictive of specific target locations. Contextual cuing for these configurations was compared to that for a set of novel repeated configurations that had not been pre-exposed. The results showed clear contextual cuing effects, with faster responses made to repeated configurations than random configurations. Furthermore, responses were faster to those configurations that had been preexposed on target-present trials compared to responses on novel repeated configurations. This result replicates the finding in Experiment 1 that pre-exposing a configuration facilitates subsequent contextual cuing for that configuration. However, a pre-exposure effect was not observed for those configurations pre-exposed on target-absent trials.

It is surprising that target-absent configurations did not confer a pre-exposure benefit on subsequent cuing given that these contexts were experienced for a substantially greater duration on each trial during the pre-exposure phase. Indeed, the exhaustive search that participants must perform on these configurations is likely to increase exposure to a greater spatial proportion of each search configuration across the many presentations during this phase. It might therefore be the case that the lack of a pre-exposure benefit from target-absent trials is not due to a deficit in the encoding of that configuration per se, but instead to the development of a strong association between the configuration and the terminating (spacebar) response as a result of the target-absent procedure. Indeed the significant cuing effect for preexposed target-absent configurations in the first phase suggests that participants had learnt to 
consistently press the spacebar in response to this set of repeated configurations. It is possible that this learnt response may have interfered with responding to the new target locations in the subsequent training phase. This account therefore suggests that the facilitation resulting from pre-exposure may have been in direct competition with the initial terminating response, and as a result may have masked any effect of pre-exposure on learning in the second phase. Experiments 3 and 4 explore possible accounts of why a pre-exposure effect was not observed for target-absent trials in Experiment 2.

\section{Experiment 3}

Experiment 3 used a timeout procedure for target-absent pre-exposure: participants had to wait for the task to terminate after a fixed duration on target-absent trials. During the preexposure phase participants were presented with repeated configurations on target-absent trials and experienced random configurations on both target-present trials and target-absent trials. The use of target-present trials during pre-exposure is important to maintain vigilance in the task to ensure encoding of the repeated configurations. In the subsequent training phase participants were trained in a standard contextual cuing task with only target-present trials and experienced pre-exposed and novel repeated configurations intermixed with random configurations.

\section{Method}

Participants. Forty participants from University College London participated in exchange for $£ 3-£ 4$ depending on their performance, using the same criteria as in Experiment 2. The experiment lasted for approximately 30 minutes.

Apparatus. The materials and apparatus were identical to Experiment 2. 
Design. Experiment 3 examined only pre-exposure on target-absent trials. Configurations were created in the same manner as for Experiment 2. Four repeated configurations were preexposed in the first phase. A set of four targets (one in each quadrant of the display) were used for these configurations in the second phase of the experiment. Two more sets of four targets (one in each quadrant) were used for novel repeated configurations and random configurations.

Procedure. The procedure was similar to that of Experiment 2 with the following changes. The pre-exposure phase consisted of 24 blocks of 20 trials. Within each block, each of the four target-absent repeated configurations was presented once. These were intermixed with 4 random target-absent configurations and 12 random target-present configurations. During this phase trials timed-out after $2000 \mathrm{~ms}$ for target-absent trials. The subsequent training phase consisted of 12 blocks of 12 trials. Each of the four configurations from each set of repeated configurations (pre-exposed and novel) was presented once and intermixed with four random configurations. The training phase consisted only of target-present trials.

\section{Results}

Three participants produced error rates on target-present trials that were greater than two SDs above the mean error rate of the sample. One additional participant produced a mean RT that was more than two SDs below the mean of the sample. These four participants were not included in the final analysis.

The criteria used in Experiment 1 were applied for data exclusions. Data were combined into two-block averages for purposes of data presentation and analysis. Figure $3 \mathrm{~A}$ shows the accuracy across the pre-exposure phase. Accuracy was very high for target-absent trials but there were no differences in the accuracy for repeated and random configurations, 
$t(35)=1.00, p=.32$. Accuracy on target-present trials was initially worse than that on targetabsent trials but reached comparable levels by the end of the phase. A one-way ANOVA found that RTs on target-present trials decreased steadily across the course of the preexposure phase, $F(11,385)=28.81, p<.001$.

Figure 3B shows RT data during the training phase. It appears that a contextual cuing effect emerged during training, with a numerically larger effect occurring for the novel repeated configurations. These data were subjected to a repeated measures ANOVA with factors of configuration (pre-exposed repeated, novel repeated, and random) and block, which revealed a marginally significant effect of configuration, $F(2,70)=2.69, p=.08$, a significant effect of block, $F(5,175)=18.95, p<.001$, and a significant interaction between configuration and block, $F(10,350)=1.96, p<.05$. Separate two-way ANOVAs provided pairwise comparisons for the levels of the configuration variable. Contrasting the two repeated configurations found no effect of configuration, $F(1,35)=1.30, p=.26$, a main effect of block, $F(5,175)=16.43, p<.001$, but no interaction between these factors, $F(5,175)$ $=1.82, p=.11$, suggesting that contextual cuing did not occur more readily for pre-exposed compared to novel repeated configurations; indeed the means are in the direction of less cuing for pre-exposed patterns. Contrasting the pre-exposed repeated configurations with random configurations revealed no main effect of configuration, $F(1,35)=1.41, p=.24$, nor a significant interaction with block, $F<1$. The main effect of block was significant, $F(5,175)$ $=12.09, p<.001$. Contrasting the novel repeated configurations with random configurations revealed a significant effect of configuration, $F(1,35)=5.30, p<.05$, a significant effect of block, $F(5,175)=15.38, p<.001$, and a significant interaction between these factors, $F(5,175)=3.32, p<.01$. 


\section{Discussion}

In Experiment 3 participants were pre-exposed to repeated configurations only on targetabsent trials. On these trials the correct response was to allow the trial to time out after two seconds. In a subsequent training phase, pre-exposed configurations were trained as predictive of target locations. However, learning about these pre-exposed configurations occurred at a similar rate to the learning that was observed for novel repeated configurations. Thus, the pattern of data was similar to that seen for the pre-exposed target-absent configurations in Experiment 2.

Whilst the aim of the pre-exposure procedure in Experiment 3 was to limit the response competition occurring on target-absent trials, it is possible that the timeout procedure led to the development of strong inhibitory response associations to pre-exposed configurations. That is, the correct response on target-absent trials was to refrain from responding, and it is possible that this inhibition may have interfered with a subsequently learnt response in the training phase.

\section{Experiment 4}

In Experiment 4 participants experienced a pre-exposure phase in which they counted the number of stimuli within the configuration. Configurations contained either eight or nine distractors and participants were asked to respond with two keys on the keyboard (odd/even). Importantly, pre-exposed configurations were presented an equal number of times with eight and nine distractors. This procedure therefore allows for pre-exposure of the repeated configurations without the development of a consistent interfering response. 


\section{Method}

Participants. Thirty-four participants from University of New South Wales participated in exchange for payment of AUS $\$ 12$. The experiment lasted for approximately 30 minutes.

Apparatus. The materials and apparatus were identical to Experiments 2 and 3. Participants used keys $\mathrm{z}$ and $\mathrm{m}$ to respond "even" or "odd" to trials in the pre-exposure phase, and the same keys were used to respond to target orientation in the training phase.

Design. Configurations were created in the same manner as for Experiments 2 and 3, except for the fact that search displays now consisted of 8 distractors. Four repeated configurations were pre-exposed in the first phase. Each configuration was presented 24 times during preexposure and was paired with the odd and even responses the same number of times (12 each). This was achieved by adding an additional random element to the configuration on half of the trials.

Procedure. The procedure was similar to that used in Experiments 2 and 3 with the following changes. The pre-exposure phase consisted of 24 blocks of 8 trials. Within each block, each of the four repeated configurations was presented once and these were intermixed with 4 random configurations. During this phase participants had to determine whether there was an odd or even number of stimuli in the display. The subsequent training phase consisted of 12 blocks of 12 trials. Each of the four configurations from each set of repeated configurations (pre-exposed and novel) was presented once and intermixed with four random configurations.

\section{Results}

Two participants produced error rates on trials in the training phase that were greater than two SDs above the mean error rate of the sample, and one additional participant produced a mean RT that was more than two SDs above the mean of the sample. These three participants 
were not included in the final analysis. The criteria used in Experiments 1-3 were applied for data exclusions. Data were combined into two-block averages for purposes of presentation and analysis.

Figure 4A shows RTs for the counting task across the 12 blocks of the pre-exposure phase. A clear difference was observed, with faster decision times to repeated configurations over random configurations. This was confirmed by a repeated measures ANOVA with factors configuration (repeated vs. random) and block, which revealed a main effect of configuration, $F(1,30)=38.06, p<.001$, and a main effect of block, $F(11,330)=8.95, p<$ .001 . The interaction between these factors was not significant, $F(11,330)=1.49, p=.13$.

Figure 4B shows RT data during the training phase. A contextual cuing effect emerged during training, with responses to repeated configurations (pre-exposed and novel) faster than those to random configurations. These data were subjected to a repeated measures ANOVA with factors configuration (pre-exposed repeated, novel repeated, and random) and block, which revealed a significant effect of configuration, $F(2,60)=10.07, p<.001$, a significant effect of block, $F(5,150)=9.55, p<.001$, and a significant interaction between configuration and block, $F(10,300)=3.82, p<.001$. Pair-wise comparisons of the different configurations revealed that responses to novel repeated configurations were faster than those to random configurations, $F(1,30)=11.25, p<.01$, a standard contextual cuing effect, and that responses to the pre-exposed configurations were also faster than those to random configurations, $F(1,30)=19.43, p<.001$. There was no difference between the responses to pre-exposed and novel repeated configurations, $F<1$, although this comparison did reveal a marginally significant interaction with the factor of block, $F(1,30)=3.13, p=.09$. Exploratory analysis revealed that there was a significant facilitation in responding to preexposed configurations (over novel repeated configurations) on the very first block of the training phase, $t(30)=3.02, p=.005$, but not in any other (when corrected for multiple 
comparisons). As in Experiments 1 and 2, the contextual cuing effect for pre-exposed repeated configurations appears to emerge rapidly in the training phase: an analysis on the first block of trials found that over the first 12 trials within that block there was no contextual cuing effect $(\mathrm{RTs}$ to repeated configurations $=863 ;$ RTs to random configurations $=871), t<$ 1, but a contextual cuing effect was present over the second set of 12 trials in block 1 (RTs to repeated configurations $=821 ;$ RTs to random configurations $=889), t(30)=2.41, p<.05$.

\section{Discussion}

In Experiment 4 participants were pre-exposed to repeated configurations in a novel counting procedure. Clearly participants were able to perform this counting task more readily for repeated configurations than for novel configurations. This is similar to the contextual cuing effect observed on target-absent trials in the pre-exposure phase of Experiment 2; possible accounts of this effect are provided in the General Discussion. In the subsequent training phase participants experienced a standard contextual cuing procedure in which they were trained with pre-exposed configurations and novel repeated configurations intermixed with random configurations. These data revealed a transient, but robust, effect of pre-exposure at the outset of this phase, representing an effect of pre-exposure on subsequent contextual cuing in configurations pre-exposed on target-absent trials. Given the absence of a target search task in this pre-exposure phase, these data provide additional support to the conclusion that the pre-exposure effect reflects learning at the level of the distractor configuration. 


\section{General Discussion}

Four experiments examined the extent to which pre-exposure of a configuration of stimuli during visual search led to the subsequent facilitation of contextual cuing within that configuration. In Experiment 1 participants were trained with repeated configurations of distractors that did not reliably signal the location of the target. During this pre-exposure phase no contextual cuing effect was observed, replicating similar findings from Chun and Jiang (Experiment 3, 1998). However, it appears that the pre-exposure of these configurations did result in them being encoded into memory, since subsequent learning about the preexposed configurations was greater than for novel repeated configurations. In Experiment 2 non-predictive configurations were pre-exposed either on trials that featured a target stimulus or on trials where the target was absent. The findings revealed a pre-exposure effect replicating that of Experiment 1 but only for those configurations pre-exposed on targetpresent trials. Experiment 3 examined whether the removal of a learnt target-absent response from the task (by using a timeout procedure, as opposed to a self-terminating procedure) would reveal a pre-exposure effect. However, there was again no evidence for a pre-exposure effect on target-absent trials. In Experiment 4 participants counted the number of distractors on the screen during the pre-exposure phase, thus removing any form of consistent motor response from the pre-exposure phase. A transient but significant facilitation in cuing was observed for the pre-exposed configurations during the subsequent training phase.

It should be noted that the major results, namely the pre-exposure effects observed in Experiments 1 and 2 with target-present configurations, were sustained across the entire training phase, whilst that observed in Experiment 4 with target-absent configurations was reflected only by a difference in performance in the first block of the training phase. Whilst further experimentation will be needed to explore these different patterns of data, we suggest at least two plausible hypotheses. Firstly, we note that the counting task in Experiment 4 led 
to substantially longer RTs in the pre-exposure phase, as a result of the more exhaustive search necessary for this task, and also resulted in a contextual cuing effect during this phase (which was not present with target-present configurations in Experiments 1 and 2). This aspect of the data suggests that participants may have become aware of the repetition of the configurations in this phase, perhaps at a much earlier stage than in the standard visual search task. This difference in explicit encoding, and the possibility of participants approaching the task in a more strategic manner, may well have resulted in rapid acquisition of the novel repeated configurations in the second phase, limiting pre-exposure to a transient effect in Experiment 4.

Pre-exposure results in acquired representations for search contexts

The results of the current experiments suggest that when participants are exposed to repeated configurations of stimuli during visual search, learning occurs not only between individual distractor elements and the target position, but also within that repeated set of distractor elements. We have tended to refer to these patterns of distractors as "configurations" and one possible means by which this learning could take place is in the formation of a distinct configural representational unit for each search context encountered in the task. Configural models of associative learning have played a dominant role as explanatory tools across a range of human and animal learning studies. According to Pearce's $(1987,1994,2002)$ model of configural learning, each presentation of a unique pattern of stimuli will result in a configural representation forming for that pattern of input to the model. This unique representational unit then acquires associative strength directly with significant events (in the current case the spatial position of the target within the display).

The results of the current experiments can certainly be accommodated within the framework of a configural model, with some assumptions. According to this account, over a 
series of presentations, the pre-exposure of a search context will result in the formation of a configural representation for that spatial arrangement of distractors. One could assume that this process is gradual and involves the stochastic sampling of the complete veridical search context. Over time, therefore, a strong representation of the complete search context will form in memory and will act as a closer match to any future samples of the search context on subsequent occasions. Thus, in the training phase, when contexts are paired consistently with target locations, each new sampling of the search context will result in a strong activation of the pre-exposed configural representation, leading to a consistent strengthening of a single configuration-target association. In contrast, for the novel repeated configurations, these configural representations will need to be established during the training phase, and hence weaker contextual cuing would be expected during the initial stages of the training phase.

This adapted configural account is certainly not the only candidate explanation for the current results. One could imagine, instead, that the representational form of the search context is entirely elemental, but involves a large network of associations forming between the distractor elements. We envisage this as a process of auto-association (e.g., McClelland \& Rumelhart, 1985) operating across repeated search contexts, such that during the preexposure phase associations are formed not just between individual distractor stimuli and the target location, but also between all the distractor stimuli within the configuration. Thus over the course of pre-exposure, this model would learn the coocurrences of distractors within repeated configurations. According to auto-associative models, when a pattern of input is presented to the model, the activation on the input layer is a product of this external input and the internally generated input from the model. Thus, pretraining allows the model to produce a more stable pattern of activation on the input layer (relative to a novel pattern), which subsequently permits stronger associations to form between distractor locations and the target position. 
We present an implementation of the auto-associative model and contrast its pattern of simulated results with those provided by the Brady and Chun (2007) model. The aim of these simulations is to account for three key aspects of the data observed regarding the robust pre-exposure effects obtained with target-present configurations in Experiments 1 and 2: 1) an absence of contextual cuing during the pre-exposure phase itself; 2) no difference in responding on repeated and random configurations at the outset of the training phase; 3) the development and sustained benefit to contextual cuing for pre-exposed over novel configurations.

Simulations with an "auto-associative" model of contextual cuing.

The auto-associative model takes as its starting point the Brady and Chun (2007) model of contextual cuing ${ }^{1}$. It uses the same structure to encode direct associations between the distractor positions and the target position, but includes an additional layer of associations that acquires associations between the individual distractor positions. Figure 5 provides an illustration of the weight structures within the model. Formally, the model sums the internally generated input for each unit, $i n_{i}$, and uses the sigmoid activation function to establish a final pattern of activation across the input units, $a_{i}$ :

$$
\begin{gathered}
i n_{i}=\left(\sum_{j} E x t_{j} \cdot A A W_{i j}\right) \\
a_{i}=1 /\left(1+e^{-i n_{i}}\right)
\end{gathered}
$$

where $E x t_{j}$ is the external input to the model on unit $j$, and $A A W_{i j}$ is the auto-associative weight between input unit $j$ and input unit $i$. The model determines the activation on the output units by a similar process:

$$
\begin{aligned}
i n_{o} & =\left(\sum_{i} a_{i} \cdot W_{o i}\right) \\
a_{o} & =1 /\left(1+e^{-i n_{0}}\right)
\end{aligned}
$$


where $W_{o i}$ is the weight between input unit $i$ and output unit $o$. From the activation across the network we take two measures of performance: the activation rank of the target unit (how active is the target relative to other units), and the mean squared error of the activation across the input units. This latter measure reflects how accurate the model is in recreating the pattern of external input from its self-generated input via the auto-associative network and therefore reflects the development of the associations between distractors.

Following the model's prediction on each trial, both sets of weights are adjusted. Weights within the auto-associative network are adjusted by:

$$
\left(\Delta A A W_{i j}\right)_{t}=\varphi \cdot\left(E x t_{i}-a_{i}\right) \cdot E x t_{j}+\theta \cdot\left(\Delta A A W_{i j}\right)_{t-1}
$$

where $\varphi$ is a parameter determining the rate of associative learning and $\theta$ is a parameter determining the contribution of momentum to weight changes. Weights for the input-output associations are adjusted by:

$$
\left(\Delta W_{o i}\right)_{t}=\varphi \cdot\left(T-a_{o}\right) \cdot a_{i}+\theta \cdot\left(\Delta W_{o i}\right)_{t-1}
$$

where $T$ is a teaching signal, taking a value of 1 if the target is presented on that output unit and 0 otherwise.

The model was trained with patterns created in an identical manner to those in Experiment 1 and with the same procedure (number of blocks, trial randomisation etc.). Results were calculated as the average performance from 100 simulated subjects, using a learning rate $(\varphi)$ of .1 and a momentum term $(\theta)$ of .1. Figure 6A shows the target rank data across the 5 blocks of pre-exposure. The model predicts a decline in the number of targets checked as a result of its learning of the most frequent target positions in the display. However, it does not predict a contextual cuing effect and therefore accurately simulates the pattern of data observed during the pre-exposure phase of Experiment 1. Figure 6B shows the target rank data across the training phase of the experiment. Whilst the model does not 
anticipate a difference in performance on pre-exposed and novel repeated configurations initially, it clearly learns more rapidly about the pre-exposed configurations across this training phase. Thus, the model accurately predicts the pre-exposure effect observed in our experiments. Figures $6 \mathrm{C}$ and $6 \mathrm{D}$ show the mean-squared error on the input units during the pre-exposure and training phases, respectively. These data show that the model rapidly acquires associations on the auto-associative layer that allow it to produce stable input representations for repeated configurations during pre-exposure. From the outset of the training phase, the model can use this stability to facilitate the development of associations between the input and output layers of the network, leading to enhanced target detection for pre-exposed configurations.

\section{Simulations with the Brady and Chun (2007) model.}

Simulations were conducted with an implementation of the Brady and Chun (2007) model using the procedure from Experiment 1. Firstly, we trained the model using the parameters specified in Brady and Chun (2007): learning rate $(\varphi)$ of .001; momentum term $(\theta)$ of .95; bottom-up activation of .1. The bottom-up activation parameter provides a boost in the activation for output units representing stimuli that are present in a given pattern, effectively suppressing locations in which stimuli are not present. Figures 7A and 7B present the simulation results across the pre-exposure and training phases, respectively. Figure 7A shows a small but highly consistent contextual cuing effect for pre-exposed configurations during the pre-exposure phase. In addition, Figure 7B shows a substantial impairment in the development of contextual cuing in the pre-exposed configurations relative to that for the novel repeated configurations. The pattern of results from this simulation does not provide a match to the ordinal pattern of data observed in Experiment 1. 
Further exploration of the parameter space revealed that increasing the bottom-up parameter to a very high value (.99) was able to reverse the pattern of results in the training phase. These simulation results are shown in Figures 7C and 7D (N.B. the scale is reduced in Figures 7C and 7D compared to Figures 7A and 7B to highlight key differences). Figure 7C shows the data from the pre-exposure phase, which again shows a contextual cuing effect for pre-exposed configurations, a pattern that was not observed in Experiments 1 or 2 for targetpresent trials (nor in Experiment 3 of Chun \& Jiang, 1998). In this simulation, pre-exposure appears to confer a short-lived facilitation on contextual cuing in the training phase, at least over the first 2-3 blocks. There are two patterns within the data, however, that suggest the effect is not driven by a facilitation in learning about pre-exposed configurations per se. Firstly, the difference between performance on pre-exposed and novel repeated configurations appears largest at the start of the training phase, whilst in Experiment 1, and in the simulation results with the auto-associative model, the pattern reflected a growth in contextual cuing across the training phase, at least until asymptotic performance was reached. In addition, it appears that the rank position of the target for pre-exposed configurations does not change between the pre-exposure and training phases. Thus the high bottom-up component leads the model to simply identify the set number of target locations, which appears to restrict the development of contextual cuing.

\section{Summary of the simulation results}

In summary, a model which includes an auto-associative layer - which allows it to learn distractor-distractor associations within the pre-exposed configurations - provides a more accurate fit to the data from Experiment 1 (and the hence the data from the target-present condition in Experiment 2) than a model that just learns distractor-target associations. The auto-associative model achieves this facilitation in contextual cuing for pre-exposed configurations by learning to produce more stable patterns of activation across the input units 
which accurately reflect the external input. This increased accuracy leads to the formation of stronger distractor-target associations once these patterns become predictive of specific target locations in the training phase.

While this new connectionist model highlights the importance of distractor-distractor learning in the contextual cuing task, it should be noted that this is clearly insufficient to drive contextual cuing alone. Indeed, the model extends rather than abandons the component processes assumed by Brady and Chun (2007). The presence of distractor-target associations is crucial for the contextual cuing effect in the model, and it is clear that distractor-target learning plays an important role in the contextual cuing effect (e.g., Conci \& Müller, 2012; Makovski \& Jiang, 2010; Manginelli \& Pollmann, 2009; Zellin, Conci, von Mühlenen, \& Müller, 2011). Furthermore, it should be noted that these associations form only part of the array of information acquired during repeated visual search. When the opportunity arises, people for example exploit high-frequency target positions at the expense of low-frequency positions (e.g., Jiang, Swallow \& Rosenbaum, 2013), anticipate an upcoming required response (e.g., Jiménez \& Vázquez, 2008; 2011), and learn relationships between object identity and target location (e.g., Endo \& Takeda, 2005). Future developments of the model will seek to accommodate such findings and could begin to translate these findings to the more complex process of real-world scene learning which is thought to include the encoding of semantic and global scene information (e.g., Brockmole, Castelhano, \& Henderson, 2006; Brockmole \& Henderson, 2006a, 2006b; Brooks, Rasmussen, \& Hollingworth, 2010).

We have provided simulations of the data from the target-present condition, but not the target-absent condition. Whilst there was evidence for a pre-exposure effect in Experiment 4 using a target-absent condition, this experiment used a unique counting procedure to reduce the impact of response-competition from target-absent responses on subsequent training (see above). We attempted only to simulate the data from conditions in 
which the standard contextual cuing procedure involving visual search for target stimuli was used throughout. It is important to note that the auto-associative model is not restricted to simulating only the pre-exposure effect in the target-present conditions, since it is the learning of co-occurring distractors in the model (the low MSE for pre-exposed patterns) that provides the simulated result; the presence of a target element is irrelevant to the development of these associations. However, any effective simulation of Experiment 4 (with the auto-associator or the Brady and Chun model) would need to make substantial changes to the way in which outcomes are encoded (e.g., to allow for even and odd responses to be made, as opposed to a prediction of the target position). Similarly, a simulation of the data from the target-absent conditions from Experiments 2 and 3 could be achieved by allowing the model to learn a no-target response in the pre-exposure phase. Given these additional complications and the clear evidence for a robust effect in the target-present conditions, it is perhaps prudent to collect more data on the pre-exposure effect in the target-absent condition before a comprehensive simulation is conducted which includes these conditions.

\section{Contextual cuing on target-absent trials}

In Experiments 2 and 4 we found that contextual cuing was observed during the pre-exposure phase for the configurations pre-exposed on target-absent trials (Experiment 3 did not permit assessment of this), whilst contextual cuing did not occur for configurations pre-exposed on target-present trials in Experiments 1 and 2. We have suggested that contextual cuing arose on target-absent trials in Experiment 2 as a result of participants learning a "no-target response" (the spacebar) to these repeated configurations. This termination of the search process can be viewed as a conditioned response elicited by the repeated configuration, much in the same way that the localization of attention to the target may be elicited by the repeated configuration as an explanation of the standard contextual cuing effect. In Experiment 4, however, there was no consistent response issued by the participant to repeated 
configurations in the pre-exposure phase. Instead, participants were asked to count the number of distractors in the configuration and report whether there was an even or odd number. Given that the configuration contained an even number of distractors on half of the trials and an odd number on the remaining trials, it seems unlikely that the contextual cuing effect occurred as a result of the learning of a consistent conditioned motor response. What seems perhaps more likely is that pre-exposure allowed participants to rapidly identify the configuration of distractors (or parts of the configuration), perhaps avoiding the need to attend to each distractor via a serial search process. This is a somewhat speculative account of the effect in the pre-exposure phase of Experiment 4 and further experimental work will be needed to examine this account in more detail. However, it is worth noting that this rapid detection of the pre-exposed configuration is in-keeping with the auto-associative model of contextual cuing. That is, by learning the distractor-distractor associations within a repeated configuration, the model is able to "retrieve" from auto-associative memory the whole configuration on the basis of a partial sampling of the pattern. Thus the model would anticipate enhanced pattern completion, which may assist in the counting task used in the preexposure phase of Experiment 4.

Finally, we note that whilst we have provided an account of the contextual cuing effect observed during pre-exposure in Experiments 2 and 4, this pattern of data is at odds with recent studies conducted by Kunar and Wolfe (2011). Of their five reported experiments, their Experiment 1 is the closest match to Experiment 2 of the current article. Twelve participants received a mixture of target-present and target-absent trials. Participants had to simply indicate whether a target was present or absent. Twelve repeated patterns were used (6 paired with a target in a particular location; 6 presented consistently with no target) and targets could appear in one of 12 locations. They also presented their repeated contexts a similar number of times (30) as we did in the pre-exposure phase of Experiment 2 (24). 
Whilst the procedure of this experiment is remarkably similar to our pre-exposure procedure in Experiment 2, the results are not: Kunar and Wolfe observed a contextual cuing effect on target-present but not on target-absent trials. This latter finding is at odds with Experiment 2 in which we observed a robust contextual cuing effect during the pre-exposure phase with target-absent trials. It is possible that the source of this discrepancy is the combined training of target-predictive configurations: in our Experiment 2 target-present repeated configurations were non-predictive of target location, while in Kunar and Wolfe's experiment they were predictive. If we imagine there is a limit to the number of context-target associations that can be acquired during contextual cuing, then perhaps in Kunar and Wolfe's experiment the simultaneous training of context-target pairings in one set restricted the development of the context-no target associations in the other. In contrast, the non-predictive contexts in the current Experiment 2 would not have exhausted such a limit. Of course, this account is purely speculative; understanding why we obtained a cuing effect with targetabsent trials while Kunar and Wolfe obtained a null result in their experiment will certainly be a theoretically interesting avenue for future experimental work.

\section{Conclusion}

In conclusion, we have demonstrated a novel pre-exposure effect in contextual cuing. The implication of this effect is that contextual cuing does not merely reflect associative learning between individual distractor locations and the target position. Rather, it suggests that the process of repeated visual search leads to a plethora of associative connections between elements of the search context. We have suggested one mechanism by which these associations can be acquired which we hope will facilitate future examinations of the representational content acquired during contextual cuing of visual search. 


\section{Footnote}

1. Note that Brady and Chun's (2007) model uses a fixed weight structure to modify the associative learning that takes place between target and distractor stimuli as a function of the distance between these stimuli. For simplicity, we do not employ these fixed weights in simulations with the auto-associative model, though a comprehensive model of contextual cuing would combine these. For our simulations of the Brady and Chun model we used a scaling factor of 10 - the largest parameter suggested by Brady and Chun - which allows the model to learn more about the surrounding distractor configuration. 
Beesley, T., \& Shanks, D. R. (2012). Investigating cue competition in contextual cuing of visual search. Journal of Experimental Psychology: Learning, Memory, and Cognition, 38, 709-25.

Bennett, I. J., Barnes, K. A., Howard, J. H., \& Howard, D. V. (2009). An abbreviated implicit spatial context learning task that yields greater learning. Behavior Research Methods, $41,391-5$.

Brady, T. F., \& Chun, M. M. (2007). Spatial Constraints on Learning in Visual Search: Modeling Contextual Cuing. Journal of Experimental Psychology: Human Perception and Performance, 33, 798 - 815.

Brockmole, J. R., Castelhano, M. S., \& Henderson, J. M. (2006). Contextual cueing in naturalistic scenes: Global and local contexts. Journal of Experimental Psychology: Learning, Memory, and Cognition, 32, 699-706.

Brockmole, J. R., \& Henderson, J. M. (2006a). Recognition and attention guidance during contextual cueing in real-world scenes: evidence from eye movements. The Quarterly Journal of Experimental Psychology, 59, 1177-87.

Brockmole, J. R., \& Henderson, J. M. (2006b). Using real-world scenes as contextual cues for search. Visual Cognition, 13, 99-108.

Brooks, D. I., Rasmussen, I. P., \& Hollingworth, A. (2010). The nesting of search contexts within natural scenes: evidence from contextual cuing. Journal of Experimental Psychology: Human Perception and Performance, 36, 1406-18.

Chun, M. M., \& Jiang, Y. (1998). Contextual Cueing: Implicit Learning and Memory of Visual Context Guides Spatial Attention. Cognitive Psychology, 71, 28-71. 
Conci, M., \& Müller, H. J. (2012). Contextual learning of multiple target locations in visual search. Visual Cognition, 20, 746-770.

Conci, M., Sun, L., \& Müller, H. J. (2011). Contextual remapping in visual search after predictable target location changes. Psychological Research, 75, 279-289.

Endo, N., \& Takeda, Y. (2004). Selective learning of spatial configuration and object identity in visual search. Perception \& Psychophysics, 66, 293-302.

Jiang, Y. V, Swallow, K. M., \& Rosenbaum, G. M. (2013). Guidance of spatial attention by incidental learning and endogenous cuing. Journal of Experimental Psychology. Human Perception and Performance, 39, 285-97.

Jiang, Y., \& Wagner, L. C. (2004). What is learned in spatial contextual cuingconfiguration or individual locations? Perception \& Psychophysics, 66, 454-463.

Jiménez, L., \& Vázquez, G. A. (2011). Implicit sequence learning and contextual cueing do not compete for central cognitive resources. Journal of Experimental Psychology: Human Perception and Performance, 37, 222-235.

Kunar, M.A., Flusberg, S.J., Horowitz, T.S., \& Wolfe, J.M., (2007). Does contextual cueing guide the deployment of attention? Journal of Experimental Psychology: Human Perception and Performance, 33, 816-828

Kunar, M. a, \& Wolfe, J. M. (2011). Target absent trials in configural contextual cuing. Attention, Perception \& Psychophysics, 73, 2077-91.

Makovski, T., \& Jiang, Y. V. (2010). Contextual cost: when a visual-search target is not where it should be. The Quarterly Journal of Experimental Psychology, 63, 216-25. 
Manginelli, A. A., \& Pollmann, S. (2009). Misleading contextual cues: How do they affect visual search? Psychological Research, 73, 212-221.

McClelland, J. L., \& Rumelhart, D. E. (1985). Distributed memory and the representation of general and specific information. Journal of Experimental Psychology: General, 114, 159-188.

Olson, I. R., \& Chun, M. M. (2002). Perceptual constraints on implicit learning of spatial context. Visual Cognition, 9, 273-302.

Pearce, J.M. (1987).A model for stimulus generalization in Pavlovian conditioning. Psychological Review, 94, 61-73.

Pearce, J.M. (1994). Similarity and discrimination: A selective review and a connectionist model. Psychological Review, 101, 587-607.

Pearce, J. M. (2002). Evaluation and development of a connectionist theory of configural learning. Animal Learning \& Behavior, 30, 73-95.

Smyth, A. C., \& Shanks, D. R. (2008). Awareness in contextual cuing with extended and concurrent explicit tests. Memory \& Cognition, 36, 403-415.

Song, J., \& Jiang, Y. V. (2005). Connecting the past with the present: How do humans match an incoming visual display with visual memory? Journal of Vision, 5, 322-330.

Zellin, M., Conci, M., von Mühlenen, A., \& Müller, H. J. (2011). Two (or three) is one too many: testing the flexibility of contextual cueing with multiple target locations. Attention, Perception \& Psychophysics, 73, 2065-76. 
Zellin M., Conci M., von Mühlenen A., \& Müller H. J. (2013). Here today, gone tomorrow - adaptation to change in memory-guided visual search. PLoS ONE 8(3): e59466. doi:10.1371/journal.pone.0059466.

Zellin, M., von Mühlenen, A., Müller, H. J., \& Conci, M. (2013). Statistical learning in the past modulates contextual cueing in the future. Journal of Vision, 13, 1-14. 
Author Note

Tom Beesley and Daniel Pearson, School of Psychology, University of New South Wales, Sydney, New South Wales, Australia; Miguel A. Vadillo and David R. Shanks, Division of Psychology and Language Sciences, University College London, London, UK.

We are grateful to Brooke Hahn for her assistance in data collection. This work was supported by grant ES/J007196/1 from the Economic and Social Research Council, awarded to David R. Shanks and Tom Beesley. Correspondence concerning this article should be addressed to Tom Beesley, School of Psychology, University of New South Wales, Sydney, New South Wales, Australia, 2052. Email: t.beesley@unsw.edu.au 
Figure 1A. RTs during the pre-exposure phase of Experiment 1.

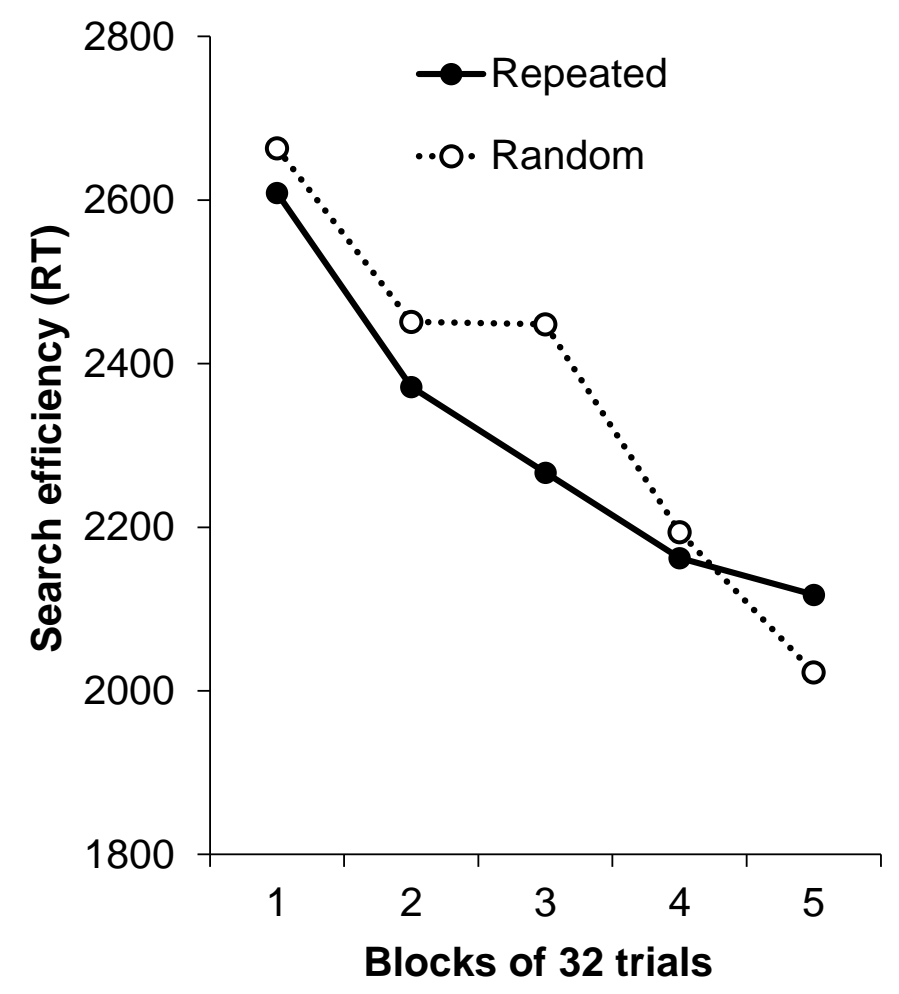

Figure 1B. RTs during the training phase of Experiment 1.

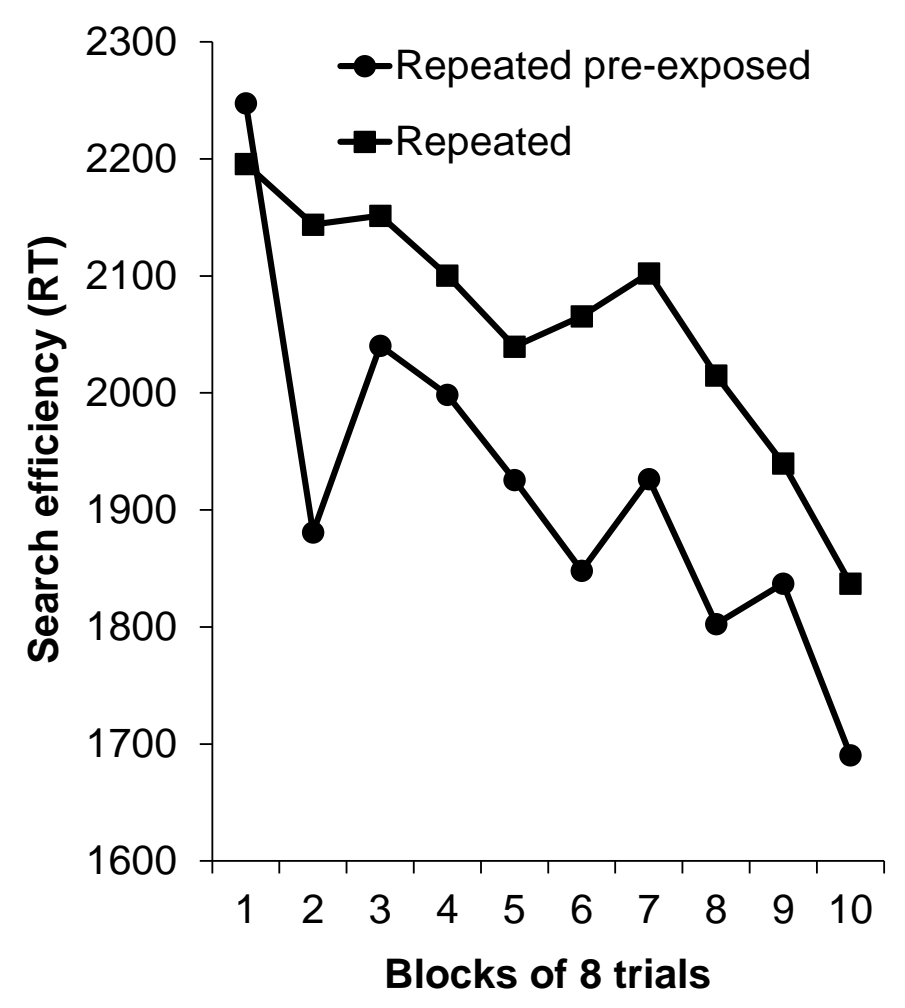


Figure 2A. RTs during the pre-exposure phase of Experiment 2.

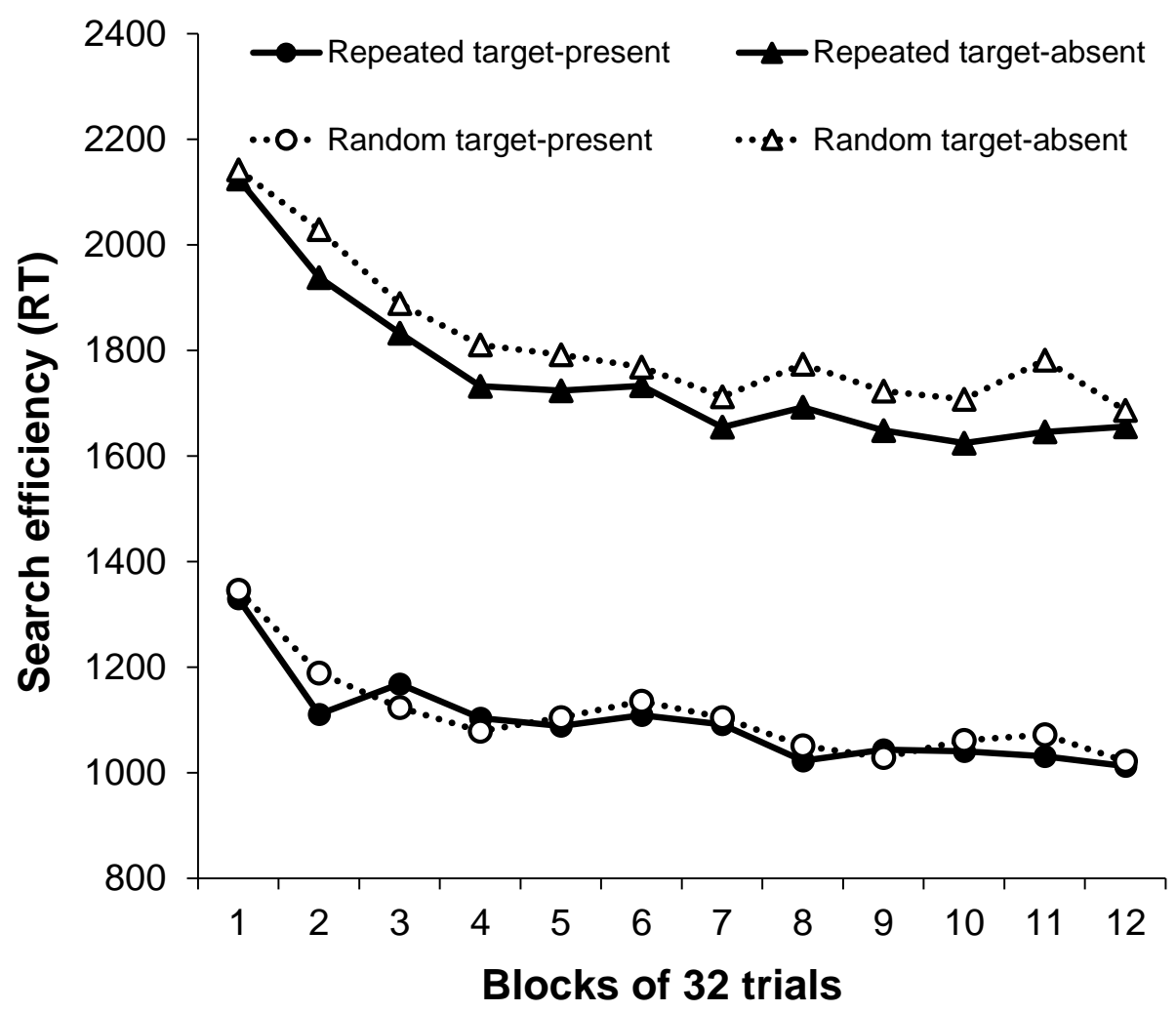

Figure 2B. RTs during the training phase of Experiment 2.

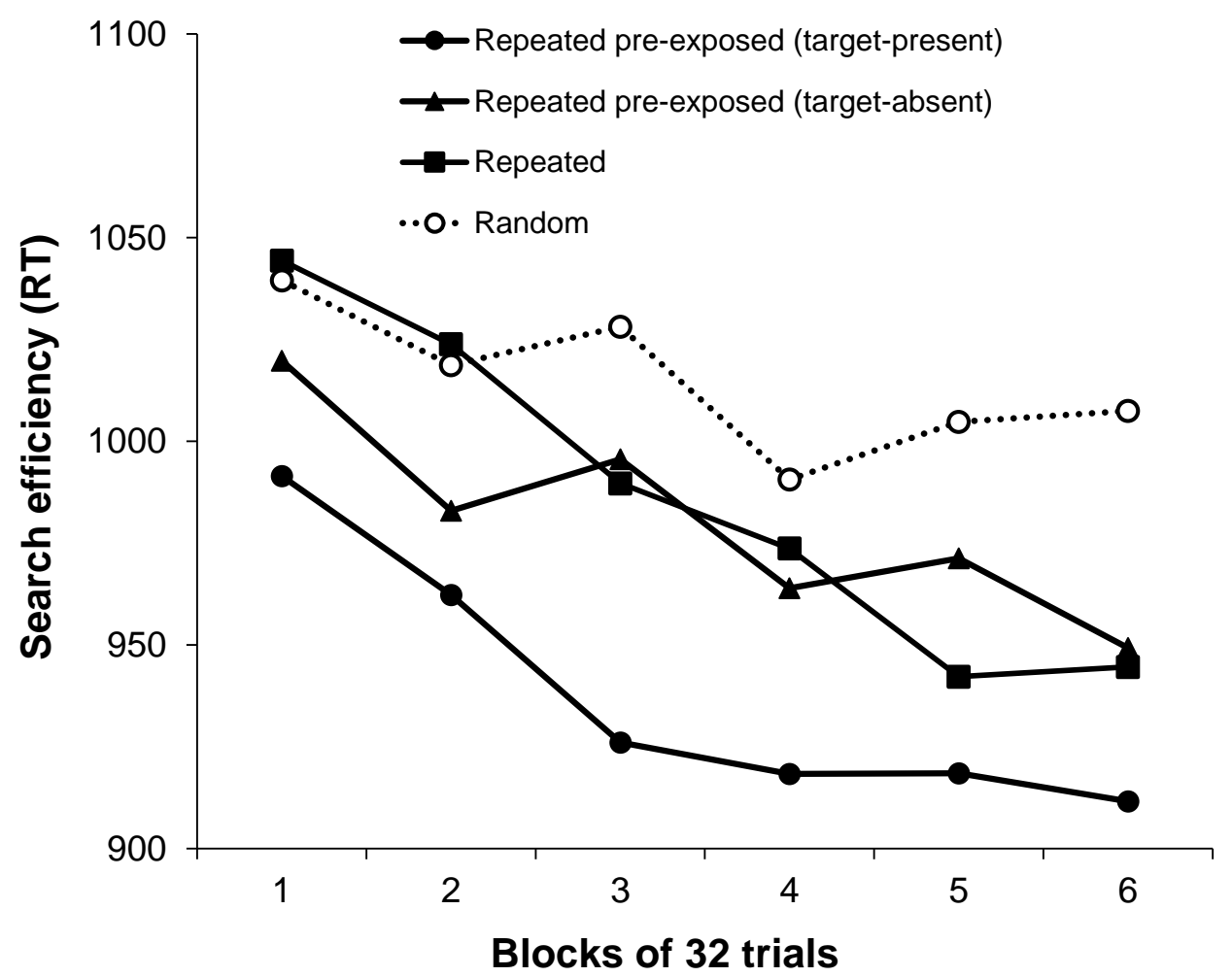


Figure 3A. Accuracy of response during the pre-exposure phase of Experiment 3.

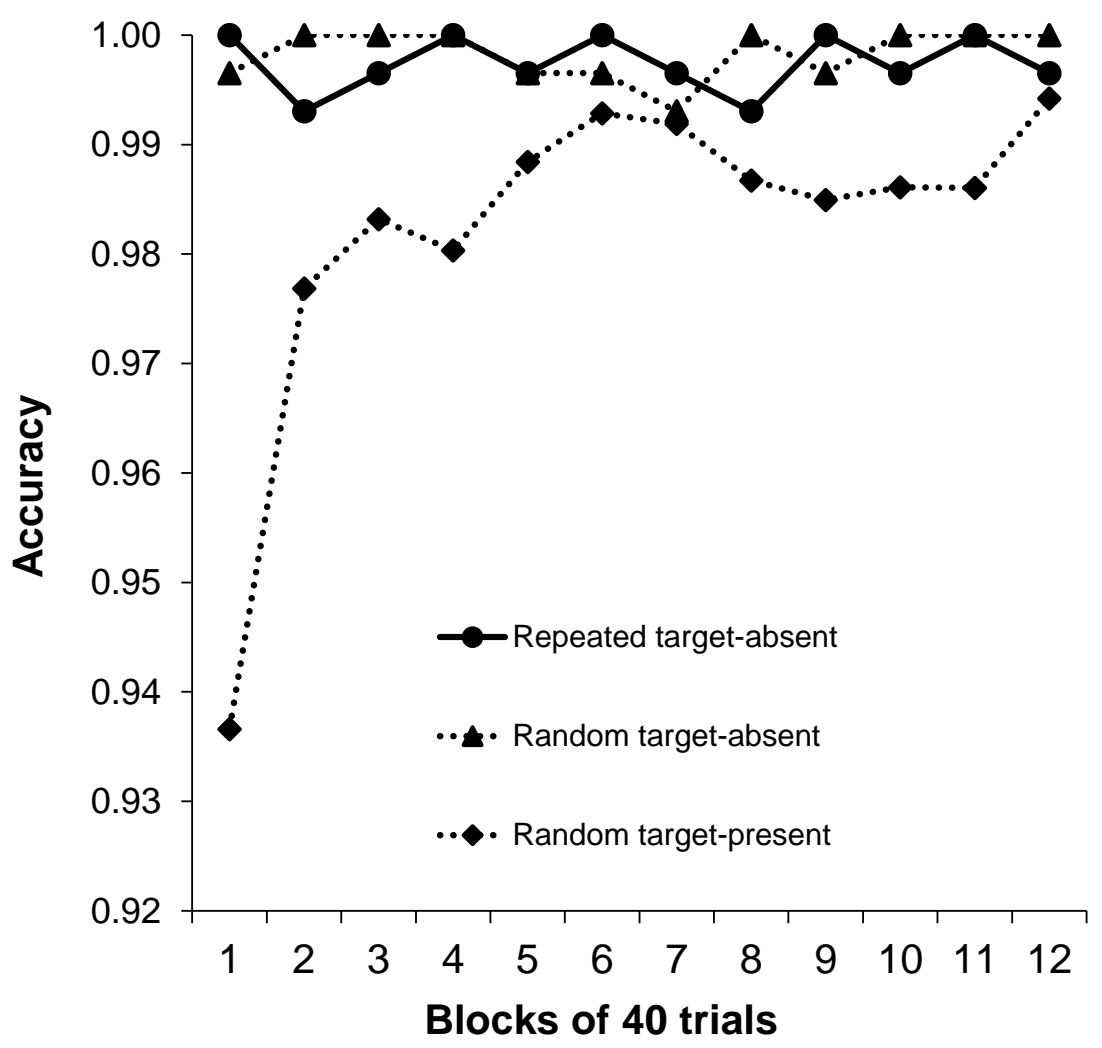

Figure 3B. RTs during the training phase of Experiment 3.

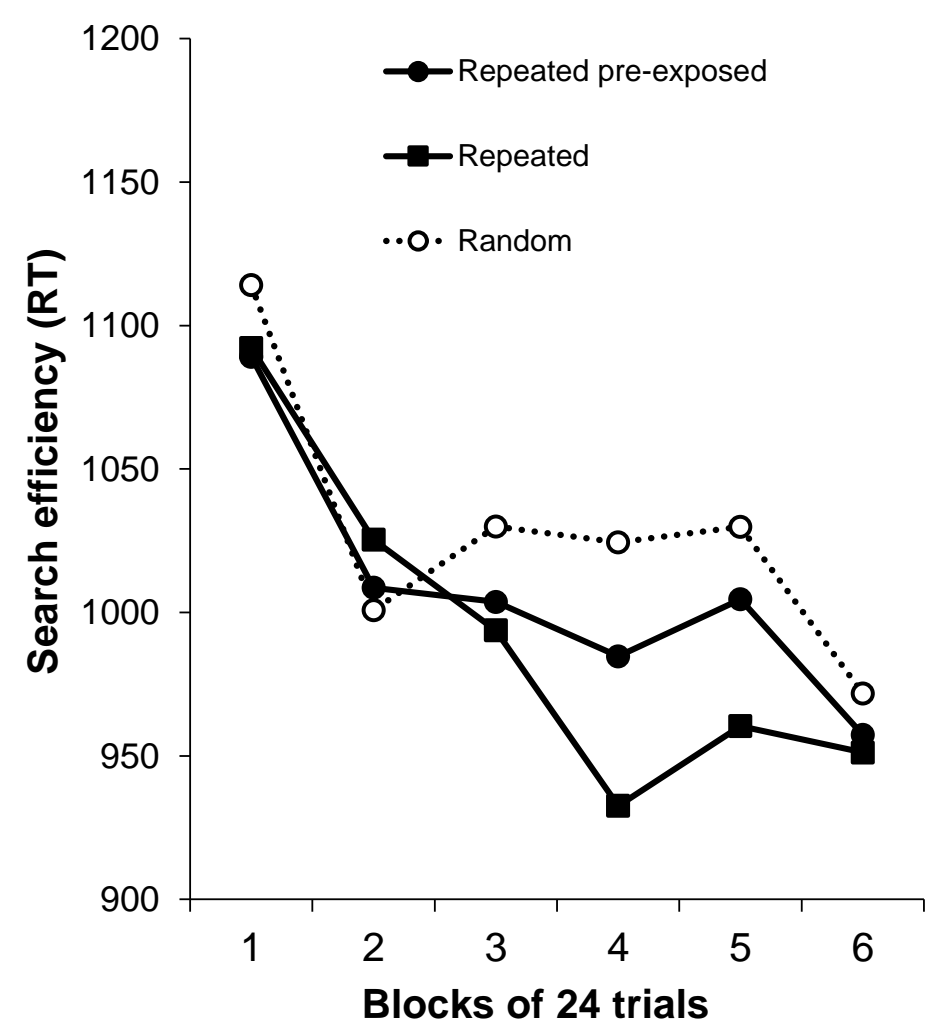


Figure 4A. RTs during the pre-exposure phase of Experiment 4.

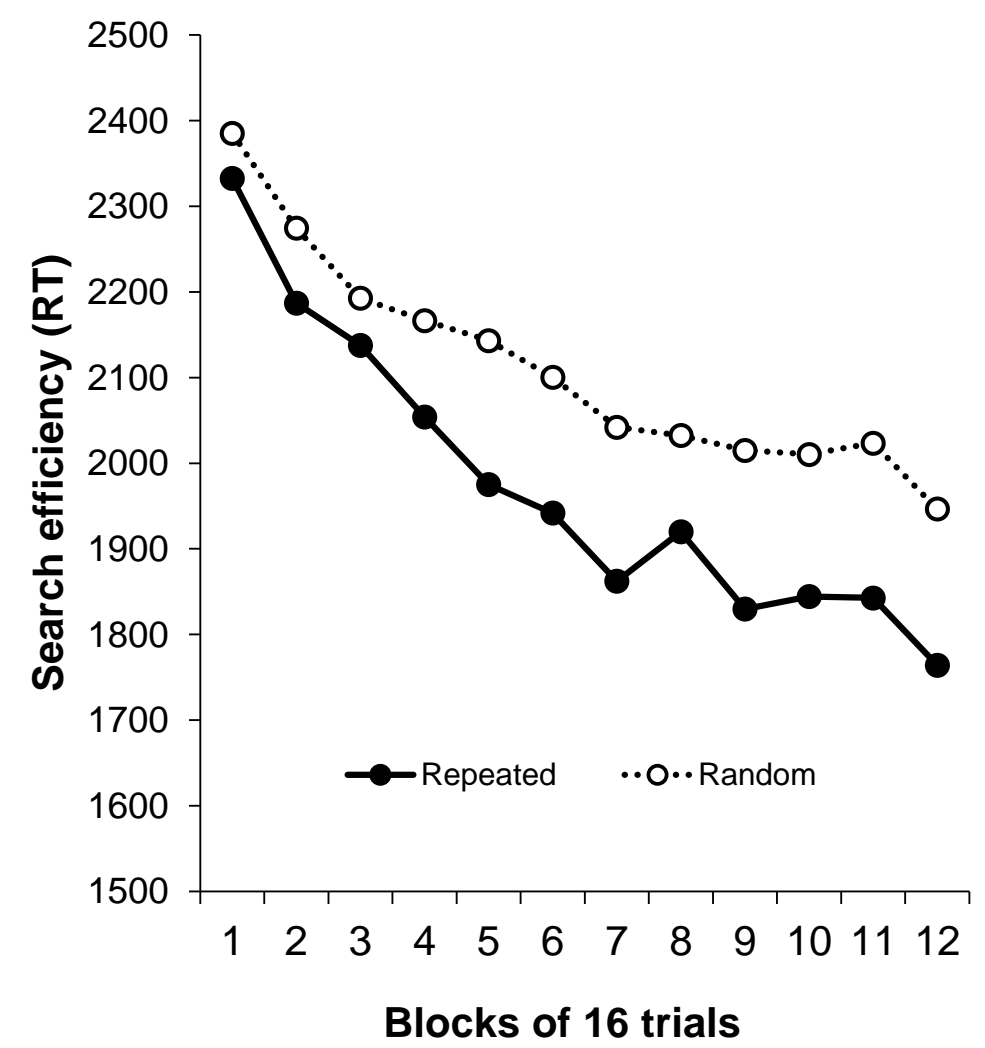

Figure 4B. RTs during the training phase of Experiment 4.

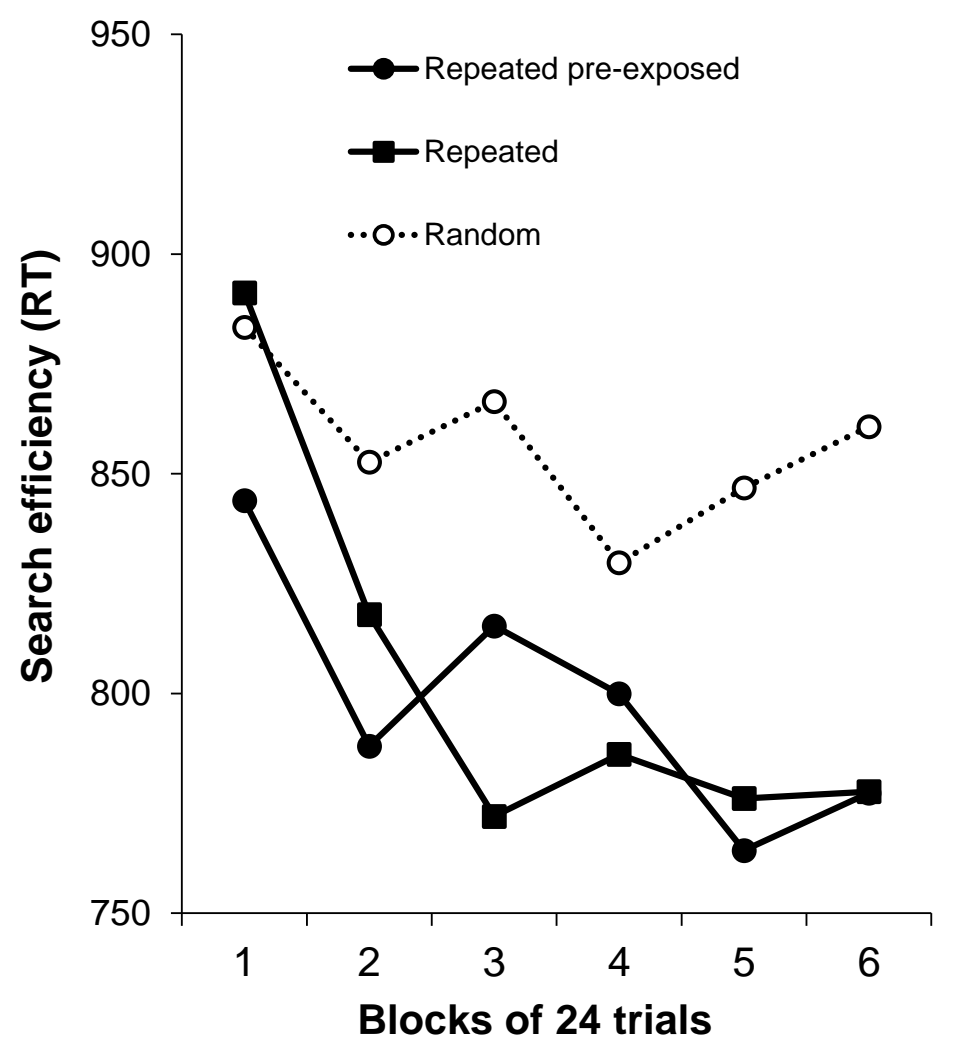


Figure 5. An auto-associative model of contextual cuing. The model features two sets of weights. Associations forming between input units $(i 1 \ldots i \mathrm{~N})$ reflect a representation of the cooccurrences of distractor stimuli within repeated patterns of context (scene memory).

Associations forming between input units and output units $(o 1 \ldots o \mathrm{~N})$ reflect representations between distractor locations and the spatial location of paired target stimuli.

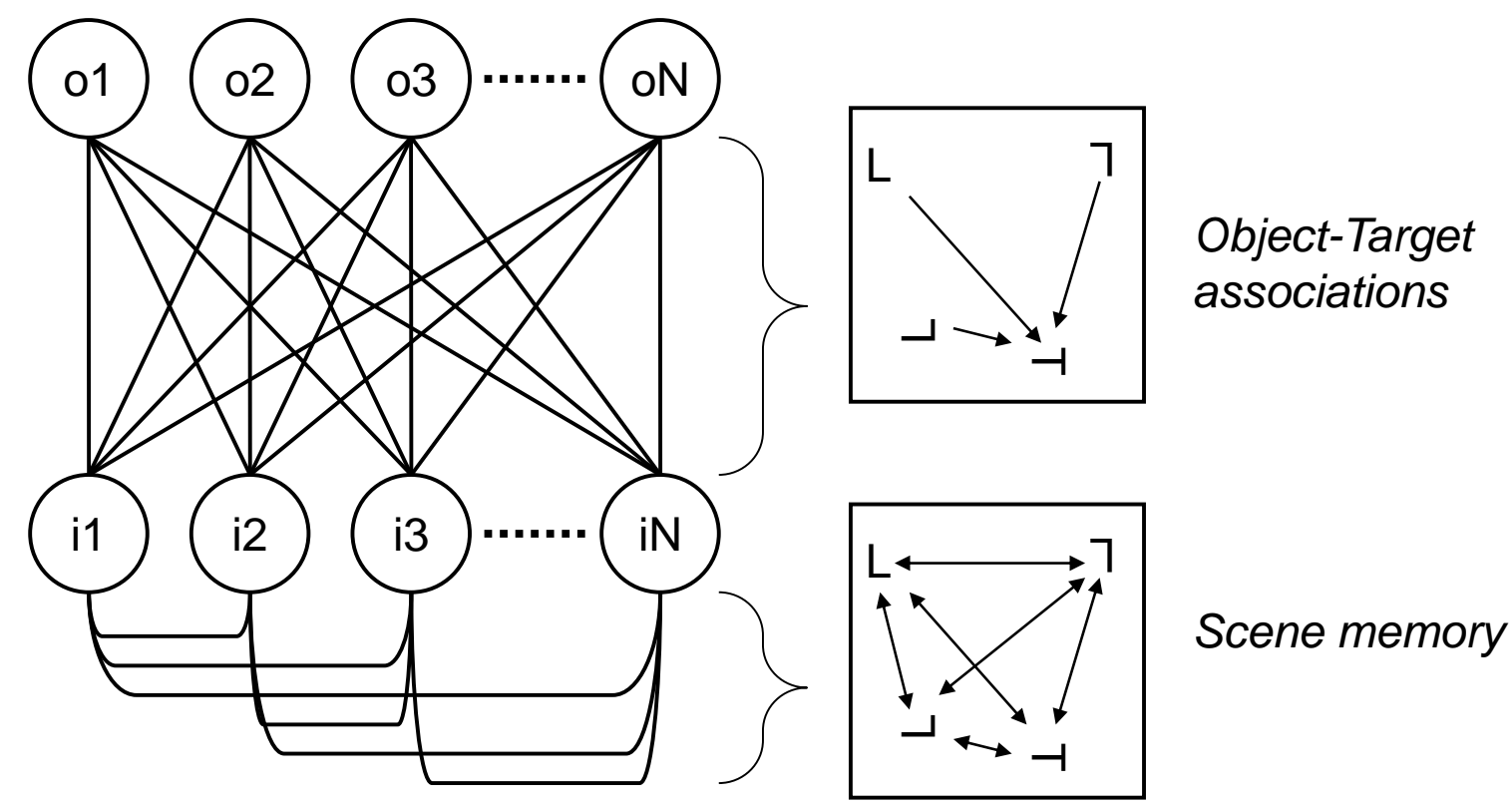


Figure 6. Simulations of the pre-exposure effect (Experiment 1) with the auto-associative network. Panels A and B show the ranked position of the activation of the output unit for the target position (amongst all output units) during the pre-exposure and training phases, respectively. Panels $C$ and $D$ show the mean squared error of the activation on the input units during the pre-exposure and training phases, respectively.

A

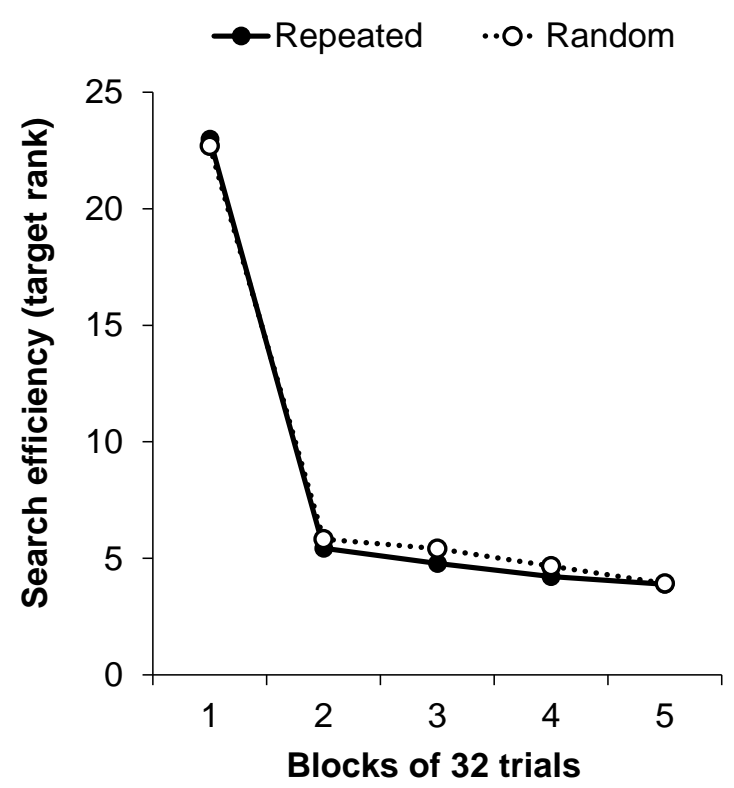

C

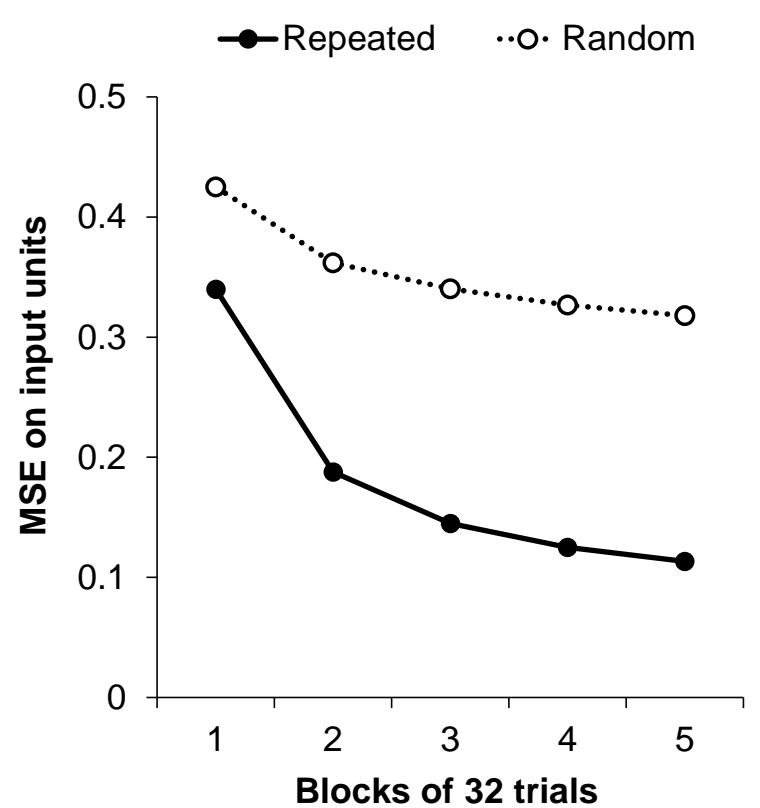

B

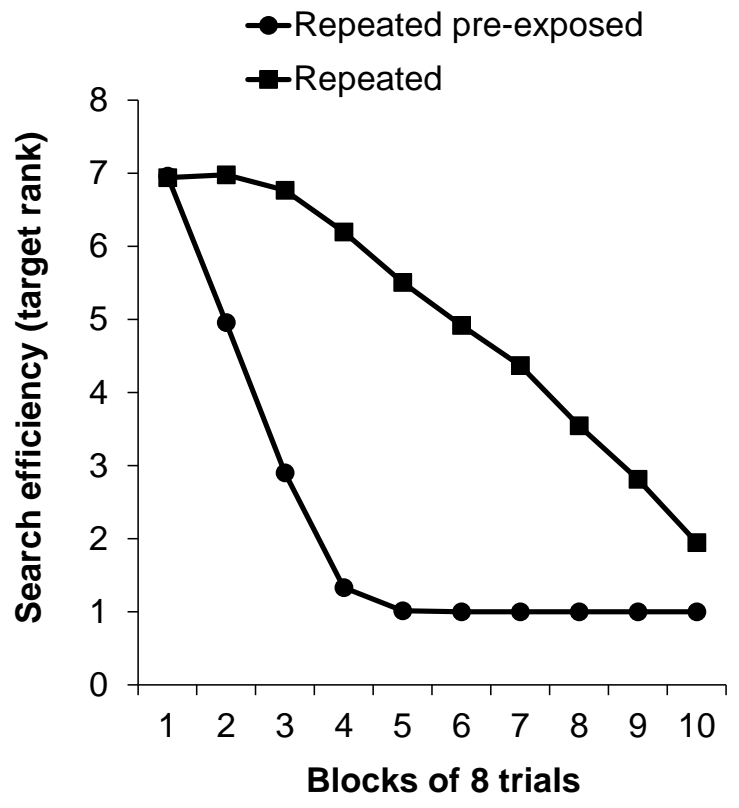

D

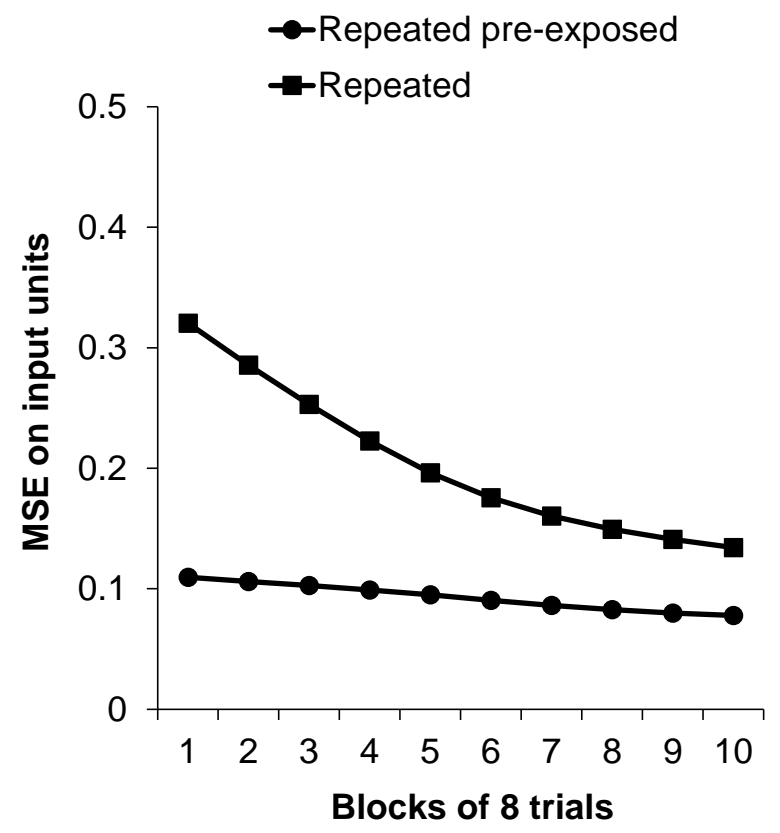


Figure 7. Simulations of the pre-exposure effect (Experiment 1) with the Brady and Chun (2007) network for two different sets of parameters (see text for details). Data show the ranked position of the activation of the output unit for the target position (amongst all output units) during the pre-exposure (panel A: simulation 1; panel C: simulation 2) and training phases (panel B: simulation 1; panel D: simulation 2).

A

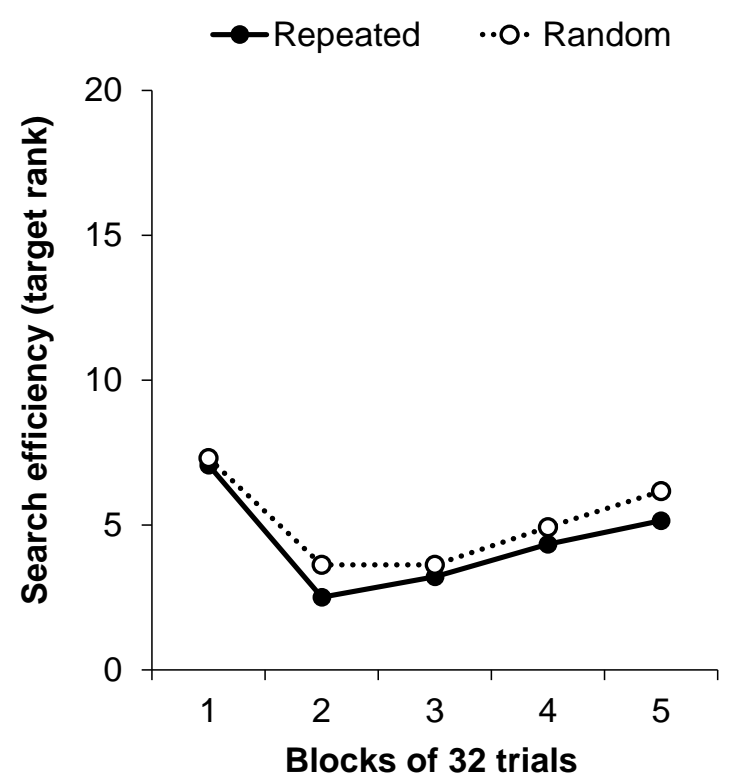

C

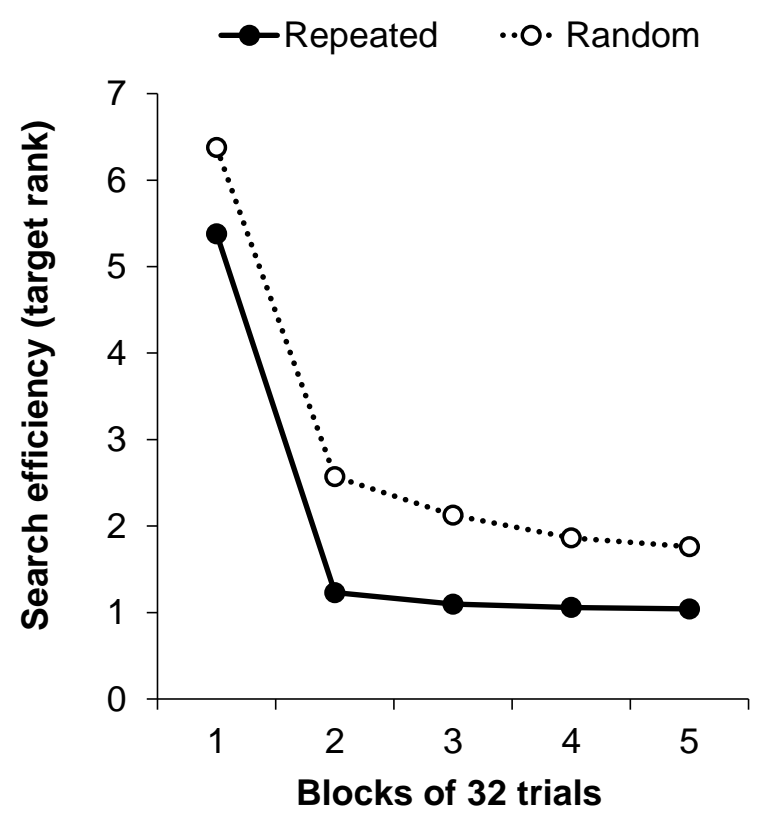

B

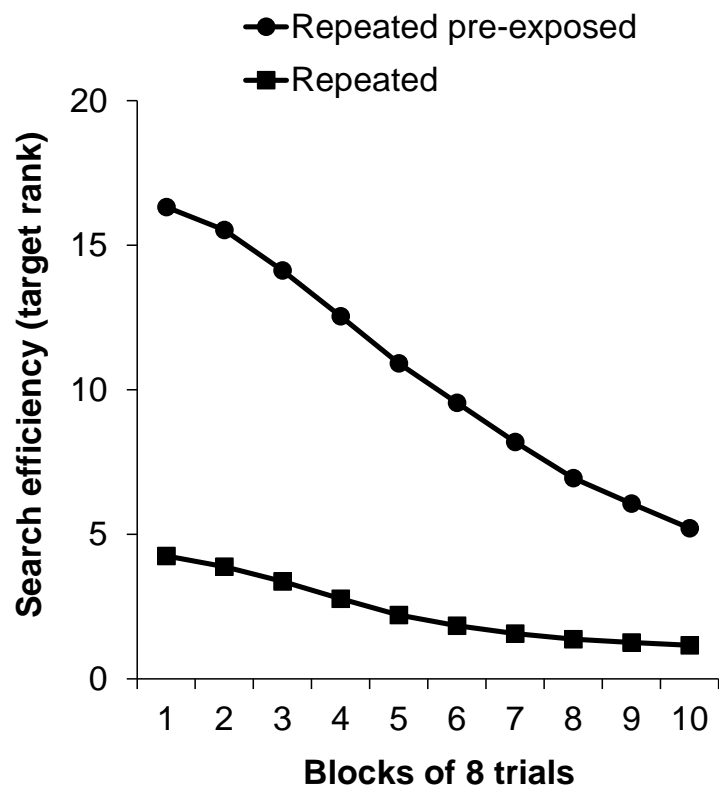

D

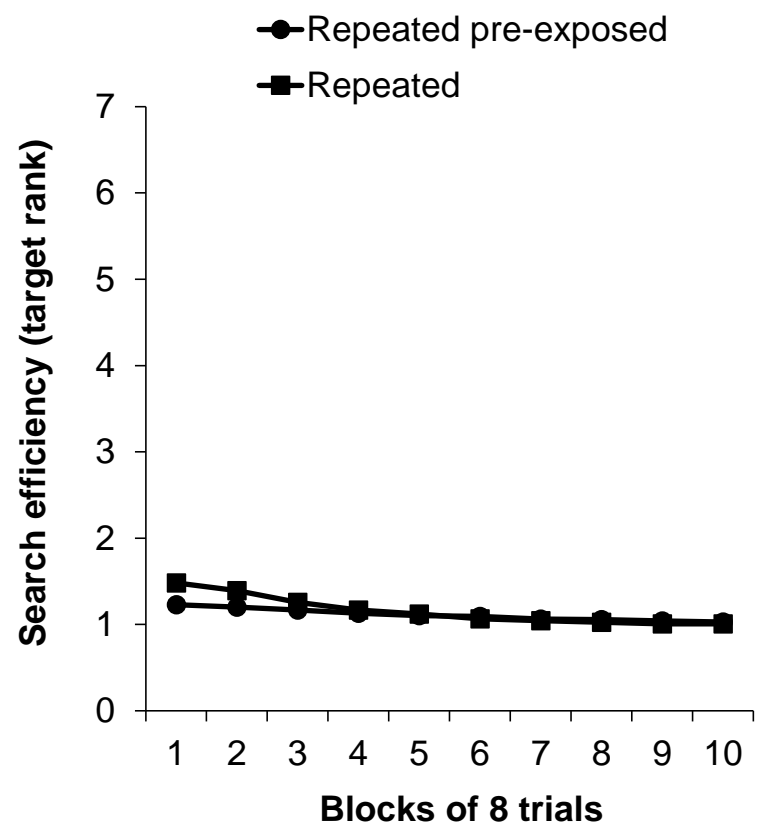

